\title{
Renormalization of the ladder light-front Bethe-Salpeter equation in the Yukawa model
}

\author{
J. H. O. Sales, ${ }^{1, *}$ T. Frederico, ${ }^{1}$ B. V. Carlson, ${ }^{1}$ and P. U. Sauer ${ }^{2}$ \\ ${ }^{1}$ Departamento de Física, Instituto Tecnológico de Aeronáutica, Centro Técnico Aeroespacial, 12.228-900 São José dos Campos, \\ São Paulo, Brazil \\ ${ }^{2}$ Institute for Theoretical Physics, University Hannover, D-30167 Hannover, Germany
}

(Received 5 July 2000; published 11 May 2001)

\begin{abstract}
The reduction of the two-fermion Bethe-Salpeter equation in the framework of light-front dynamics is studied for the Yukawa model. It yields auxiliary three-dimensional quantities for the transition matrix and the bound state. The arising effective interaction can be perturbatively expanded in powers of the coupling constant $g_{s}$ allowing a defined number of boson exchanges; it is divergent and needs renormalization; it also includes the instantaneous term of the Dirac propagator. One possible solution of the renormalization problem of the boson exchanges is shown to be provided by expanding the effective interaction beyond single boson exchange. The effective interaction in ladder approximation up to order $g_{s}^{4}$ is discussed in detail. It is shown that the effective interaction naturally yields the "box counterterm" required to be introduced ad hoc previously. The covariant results of the Bethe-Salpeter equation can be recovered from the corresponding auxiliary three-dimensional quantities.

DOI: 10.1103/PhysRevC.63.064003

PACS number(s): 12.39.Ki, 14.40.Cs, 13.40.Gp
\end{abstract}

\section{INTRODUCTION}

The Bethe-Salpeter equation (BSE) [1] describes interacting two-particle systems in the framework of a relativistic field theory. The transition matrix $T$ of two-particle scattering satisfies the inhomogeneous BSE

$$
T=V+V G_{0}^{F} T .
$$

In the above equation $G_{0}^{F}$ is the disconnected Green's function for two fermions. It is the Green's function for two noninteracting particles when self-energy contributions are neglected. The two-fermion free Green's function,

$$
G_{0}^{F}=\frac{i\left(\hat{k}_{1}+m_{1}\right)}{\hat{k}_{1}^{2}-m_{1}^{2}+i o} \frac{i\left(\hat{k}_{2}+m_{2}\right)}{\hat{k}_{2}^{2}-m_{2}^{2}+i o},
$$

can be split up according to

$$
G_{0}^{F}=\bar{G}_{0}+\Delta G_{0}^{F},
$$

where we define

$$
\begin{aligned}
& \bar{G}_{0}=\left(\hat{k}_{1 o n}+m_{1}\right)\left(\hat{k}_{2 o n}+m_{2}\right) G_{0}, \\
& G_{0}=\frac{i}{\hat{k}_{1}^{2}-m_{1}^{2}+i o} \frac{i}{\hat{k}_{2}^{2}-m_{2}^{2}+i o} .
\end{aligned}
$$

In Eqs. (2), (4), and (5), $\hat{k}_{i}^{\mu}$ is the off-mass-shell momentum operator acting on the coordinates of particle $i$ with mass $m_{i}$, the hat on the variable indicates its operator character; the light-front components are $\hat{k}_{i o n}^{-}=\left(\hat{k}_{i \perp}^{2}+m_{1}^{2}\right) / \hat{k}_{i}^{+}$, and $\hat{k}_{i}^{+}$ $=\hat{k}_{i}^{0}+\hat{k}_{i}^{3}$. In Eq. (3) $\bar{G}_{0}$ is an auxiliary fermion operator in

\footnotetext{
*Present address: Instituto de Física Teórica, Universidade Estadual Paulista, 01405-900 São Paulo, Brazil.
}

which the spin-dependent part is formed from on-mass shell momenta and $G_{0}$ is the covariant bosonlike Green's function, whereas $G_{0}^{F}$ contains all particular divergences and subtleties connected with fermion motion. In light-front coordinates, $\Delta G_{0}^{F}$ takes the form

$$
\Delta G_{0}^{F}=\frac{\gamma_{1}^{+}}{2 \hat{k}_{1}^{+}} \frac{\hat{k}_{2 o n}+m_{2}}{\hat{k}_{2}^{2}-m_{2}^{2}+i o}+\frac{\hat{k}_{1 o n}+m_{1}}{\hat{k}_{1}^{2}-m_{1}^{2}+i o} \frac{\gamma_{2}^{+}}{2 \hat{k}_{2}^{+}}+\frac{\gamma_{1}^{+}}{2 \hat{k}_{1}^{+}} \frac{\gamma_{2}^{+}}{2 \hat{k}_{2}^{+}}
$$

and carries the instantaneous part of the fermion propagators in light-front time; it yields singular results under $k_{1}^{-}$integration.

The inhomogeneous term $V$ in Eq. (1) is the complete interaction, irreducible with respect to two-particle propagation. The two-particle bound state with total four-momentum $K_{B}, K_{B}^{2}=M_{B}^{2}$, is characterized by the vertex $\mid \Gamma$ ) at the bound-state pole of $T$, which is a solution of the homogeneous BSE

$$
\left.\mid \Gamma)=V G_{0}^{F} \mid \Gamma\right),
$$

related to the Bethe-Salpeter amplitude of the bound state by

$$
\left.\mid \Psi)=G_{0}^{F} \mid \Gamma\right) .
$$

For convenience we use the bra-ket notation with round brackets to represent functions which can be written in either momentum or coordinate spaces. The vertex function $\mid \Gamma$ ) and Bethe-Salpeter amplitude $\mid \Psi$ ) have the full four dimensional dependence on the coordinates of both particle. The normalization condition has to be defined to determine $\mid \Psi$ ) in full from the solution of Eq. (7). The operators $\mathcal{O}_{\alpha}=T$, $G_{0}$, or $V$, as well as $\left.\mid \Psi\right)$ and $\mid \Gamma$ ) carry a four-dimensional $\delta$ function in momentum space due to the conservation of the total two-particle four-momentum $K$ in Eqs. (1) and (7):

$$
\left(K^{\prime}\left|\mathcal{O}_{\alpha}\right| K\right)=\delta\left(K^{\prime}-K\right) \mathcal{O}_{\alpha}(K),
$$




$$
\begin{gathered}
\left(K^{\prime} \mid \Psi\right)=\delta\left(K^{\prime}-K_{B}\right)\left|\Psi_{B}\right\rangle, \\
\left(K^{\prime} \mid \Gamma\right)=\delta\left(K^{\prime}-K_{B}\right)\left|\Gamma_{B}\right\rangle .
\end{gathered}
$$

The reduced quantities $\left|\Psi_{B}\right\rangle,\left|\Gamma_{B}\right\rangle$ and the $\mathcal{O}_{\alpha}(K)$ are functions of the internal variables expressed in terms of the fourdimensional momentum $k^{\mu}$ or coordinate $x^{\mu}$ and depend parametrically on $K$. They satisfy Eqs. (1) and (7) in a corresponding fashion.

The field theoretic scattering amplitude and the bound state vertex function are the solutions of the BSEs (1) and (7). However, the solution of the BSE constitutes a difficult calculational task for any realistic field theory. In practical calculations, the driving term $V(K)$ has to be truncated to low orders of particle exchange [2-7]. In bosonic models, the issue of the convergence of the truncation to low orders of intermediate particle propagation has been studied recently in the two boson bound system [8-10] as well as in the scattering [11]. In fermionic models, the papers by Fuda [12] discuss one-boson exchange models in the ladder approximation in both light-front and instant-form dynamics without emphasis, however, to the underlying field-theoretic framework. The field theoretic approach in the light-front is also being used to describe finite nuclei [13] and nuclear matter with nucleon-nucleon correlations obtained at the level of the one boson exchange approximation [14].

In this work, we consider the two fermion system in the light front with one-boson exchange in the $(3+1)$ Yukawa model, for which the interaction Lagrangian density is given by

$$
\mathcal{L}_{I}=g_{S} \bar{\Psi} \Psi \sigma
$$

The fermion corresponds to the field $\Psi$ with rest masses $m$ and the exchanged boson to the field $\sigma$ with mass $\mu$. The coupling constant is $g_{S}$.

The light-front Tamm-Dancoff approximation, proposed in Ref. [4], corresponds to the truncation of the light-front Fock space, where the light-front Hamiltonian is diagonalized. In the one-boson-exchange aproximation of the twofermion bound state, the wave function has components only in the two-fermion and in the two-fermion plus one-boson sectors of the Fock space. The coupled equations in the Fock space can be reduced to a two-fermion Bethe-Salpeter equation by writing the three particle component of the wave function in terms of the projection of the wave function in the two-fermion sector. In this case, the kernel of the reduced Bethe-Salpeter equation contains the virtual state threeparticle propagation, which besides the fermion self-energy includes the one-boson-exchange interaction in the light front.

The kernel of the light-front Tamm-Dancoff [4] reduced Bethe-Salpeter equation for the vertex function for the oneboson-exchange interaction in ladder approximation, ignoring self-energy contributions, has a divergence problem, since the kernel becomes independent of the integrated transverse momentum when it goes to infinity [15]. The asymptotic behavior of the two-fermion bound state wave function does not render the integration over the internal transverse momentum finite for some of its spin components [15]. To solve this problem in the $(3+1)$ Yukawa model in the lowest order of the light-front Tamm-Dancoff approximation, without self-energy terms, the introduction of a counterterm to renormalize the integral equation was proposed in Ref. [5]. The transverse momentum cutoff dependence of the bound-state mass is reduced or vanishes depending on the procedure chosen to renormalize the lightfront integral equation [5]. A lowest order perturbative analysis performed in Ref. [5], provides the so-called "box counterterm', which is nonlocal, and reduced considerably the cutoff dependence of the bound-state mass. Reference [5] also derives an additional asymptotic counterterm which completely removes the cutoff dependence.

In the present work, we are concerned with the origin of the perturbative counterterms of the light-front ladder BetheSalpeter equation for the Yukawa model. We will show that using the systematic expansion of the Bethe-Salpeter equation in the light-front developed in Ref. [8], the kernel of the auxiliary integral equation, expanded up to order $g_{S}^{4}$, naturally yields the "box counterterm" [5] and a well defined finite part. We derive the "box counterterm" from the contribution of the intermediate state light-front virtual propagation of four particles (two sigmas and two fermions), including the instantaneous terms of the fermion propagators in order $g_{S}^{4}$. Although, we have exemplified the systematic expansion of the kernel of the projected Bethe-Salpeter equation in the light-front up to order $g_{S}^{4}$, the construction of the kernel of the auxiliary integral equation can be performed, in principle, to any desired order in the perturbative expansion. It is remarkable, that the cancellation of singularities, exemplified at order $g_{S}^{4}$, occurs at all orders. This is because, the perturbative expansion of the light-front scattering amplitude in powers of the coupling constant, obtained from the lightfront $T$-matrix equation with the kernel calculated up to the same order, necessarily reproduces the perturbative covariant ladder scattering amplitude at that order of $g_{S}$. Consequently the ladder approximation to the Bethe-Salpeter equation in the light-front does not need a new class of couterterms. To the best of the authors' knowledge, no other work has achieved such an explicit systematic reduction of a given four-dimensional equation of a fermionic model to the light front.

Section II discusses three-dimensional auxiliary quantities from which the covariant solutions of the BSE can be obtained. The auxiliary quantities are operators and functions defined on the light front. Section III gives our theoretical apparatus in full for the Yukawa model. Sections II and III follow closely our previous paper on the bosonic model [8] with the aim of presenting the underlying formalism of this work in complete form. Section IV, which is the central part of the present work, discusses the effective threedimensional interaction in second order of the coupling constant $g_{s}$, and shows that the perturbative "box couterterm", appears naturally in the systematic expansion of the effective interaction. Our conclusions are given in Sec. V.

\section{TWO-PARTICLE AUXILIARY FREE GREEN'S FUNCTION $\widetilde{G}_{0}(K)$}

The transition matrix $T(K)$ and the bound-state amplitude $\left|\Psi_{B}\right\rangle$ of the covariant BSE can be obtained with the help of 
a convenient auxiliary Green's function $\widetilde{G}_{0}(K)$ [16], which will be chosen to include explicitly the propagation of the two-fermion system between two light-front hyperplanes of $x^{+}=x^{0}+x^{3}=$ const, as precisely defined by Eq. (30) and discussed at the end of this section. This and the following section generalize the discussion presented in Ref. [8] in the context of bosons to fermions. According to Ref. [16], we have

$$
\begin{gathered}
T(K)=W(K)+W(K) \widetilde{G}_{0}(K) T(K), \\
\left|\Gamma_{B}\right\rangle=W\left(K_{B}\right) \widetilde{G}_{0}\left(K_{B}\right)\left|\Gamma_{B}\right\rangle, \\
\left|\Psi_{B}\right\rangle=G_{0}^{F}(K)\left|\Gamma_{B}\right\rangle,
\end{gathered}
$$

where the driving term $V(K)$ is changed to $W(K)$, given by $V(K)$ according to the integral equation

$$
W(K)=V(K)+V(K)\left[G_{0}^{F}(K)-\widetilde{G}_{0}(K)\right] W(K) .
$$

The normalization condition for the bound-state BetheSalpeter amplitude $\left|\Psi_{B}\right\rangle$ is

$$
\begin{aligned}
& \lim _{K^{2} \rightarrow K_{B}^{2}}\left\langle\Psi_{B}\left|\frac{G_{0}^{F}(K)^{-1}-G_{0}^{F}\left(K_{B}\right)^{-1}}{K^{2}-K_{B}^{2}}-\frac{V(K)-V\left(K_{B}\right)}{K^{2}-K_{B}^{2}}\right| \Psi_{B}\right\rangle \\
& \quad=1 .
\end{aligned}
$$

It involves the original driving term $V(K)$ [17]. As was the case for the bosonic light-front propagator in the ladder ap- proximation [8], the aim here is to choose $\widetilde{G}_{0}(K)$ such that the integral equation (16) does not have to be solved in full, but that a few terms of the infinite series

$$
\begin{gathered}
W(K)=V(K) \sum_{n=0}^{\infty}\left[\left(G_{0}^{F}(K)-\widetilde{G}_{0}(K)\right) V(K)\right]^{n}, \\
W(K)=V(K)+V(K)\left(G_{0}^{F}(K)-\widetilde{G}_{0}(K)\right) V(K)+\cdots
\end{gathered}
$$

will be sufficient for a converged solution of the BSE. The auxiliary Green's function $\widetilde{G}_{0}(K)$ remains a fourdimensional one, but its choice may sacrifice the covariance which $G_{0}^{F}(K)$ possesses.

The dynamics of the interacting two-particle system can be fully described by its propagation between hyperplanes, the hyperplanes $x^{0}=$ const in instant-form dynamics, the hyperplanes $x^{+}=x^{0}+x^{3}=$ const in light-front dynamics [18]. The null-plane defined by $x^{+}=0$ is special since it is left invariant under seven kinematical boosts, while the $x^{+}$ $=$ const hyperplanes scale under light-front boosts. In contrast, the free Green's function of the BSE depends on the individual times $x_{i}^{0}$ or on the individual light-front times $x_{i}^{+}$.

According to Eq. (4) the propagating part $\bar{G}_{0}$ of the free Green's function in light-front coordinates, $k_{i}=\left(k_{i}^{-}=k_{i}^{0}\right.$ $\left.-k_{i}^{3}, k_{i}^{+}=k_{i}^{0}+k_{i}^{3}, \vec{k}_{\perp}\right)$

$$
\begin{aligned}
\left\langle x_{1}^{\prime+} x_{2}^{\prime+}\left|\bar{G}_{0}\right| x_{1}^{+} x_{2}^{+}\right\rangle= & -\frac{1}{(2 \pi)^{2}} \int d k_{1}^{-} d K^{-} e^{-(i / 2) k_{1}^{-}\left(x_{1}^{\prime+}-x_{2}^{\prime+}-x_{1}^{+}+x_{2}^{+}\right)} e^{-(i / 2) K^{-}\left(x_{2}^{\prime+}-x_{2}^{+}\right)} \\
& \times \frac{\left(\hat{k}_{1 o n}+m_{1}\right)\left(\hat{k}_{2 o n}+m_{2}\right)}{\hat{k}_{1}^{+}\left(K^{+}-\hat{k}_{1}^{+}\right)\left(k_{1}^{-}-\frac{\hat{\vec{k}}_{1 \perp}^{2}+m_{1}^{2}-i o}{\hat{k}_{1}^{+}}\right)\left(K^{-}-k_{1}^{-}-\frac{\hat{\vec{k}}_{2 \perp}^{2}+m_{2}^{2}-i o}{K^{+}-\hat{k}_{1}^{+}}\right)}
\end{aligned}
$$

(only its dependence on the individual light-front "times" $x_{i}^{+}$is made explicit), reduces, for propagation between the hyperplanes $x_{1}^{+}=x_{2}^{+}=x^{+}$and $x_{1}^{\prime+}=x_{2}^{\prime+}=x^{\prime+}$, to

$$
\begin{aligned}
\left\langle x^{\prime+} x^{\prime+}\left|\bar{G}_{0}\right| x^{+} x^{+}\right\rangle & =\int \frac{d K^{-}}{2 \pi} e^{-(i / 2) K^{-}\left(x^{\prime+}-x^{+}\right)} \int d k_{1}^{\prime-} d k_{1}^{-}\left\langle k_{1}^{\prime-}\left|\bar{G}_{0}(K)\right| k_{1}^{-}\right\rangle, \\
& \equiv \int \frac{d K^{-}}{2 \pi} e^{-(i / 2) K^{-}\left(x^{\prime+}-x^{+}\right)}\left|\bar{G}_{0}(K)\right| .
\end{aligned}
$$

In Eq. (20), the notation

$$
\left\langle k_{1}^{\prime-}\left|\bar{G}_{0}(K)\right| k_{1}^{-}\right\rangle=-\frac{\delta\left(k_{1}^{\prime-}-k_{1}^{-}\right)}{2 \pi} \frac{\left(\hat{k}_{1 o n}+m_{1}\right)\left(\hat{k}_{2 o n}+m_{2}\right)}{\hat{k}_{1}^{+}\left(K^{+}-\hat{k}_{1}^{+}\right)\left(k_{1}^{-}-\frac{\hat{\vec{k}}_{1 \perp}^{2}+m_{1}^{2}-i o}{\hat{k}_{1}^{+}}\right)\left(K^{-}-k_{1}^{-}-\frac{\hat{\vec{k}}_{2 \perp}^{2}+m_{2}^{2}-i o}{K^{+}-\hat{k}_{1}^{+}}\right)}
$$

is introduced, as well as 


$$
\begin{aligned}
\left|\bar{G}_{0}(K)\right|: & =\int d k_{1}^{\prime-} d k_{1}^{-}\left\langle k_{1}^{\prime-}\left|\bar{G}_{0}(K)\right| k_{1}^{-}\right\rangle \\
& =i \theta\left(K^{+}-\hat{k}_{1}^{+}\right) \theta\left(\hat{k}_{1}^{+}\right) \frac{\left(2 m_{1}\right)\left(2 m_{2}\right) \Lambda_{+}\left(\hat{k}_{1 o n}\right) \Lambda_{+}\left(\hat{k}_{2 o n}\right)}{\hat{k}_{1}^{+}\left(K^{+}-\hat{k}_{1}^{+}\right)\left(K^{-}-\hat{k}_{1 o n}^{-}-\hat{k}_{2 o n}^{-}+i o\right)} \\
& :=g_{0}(K),
\end{aligned}
$$

where $K^{+}>0$ can be chosen without any loss of generality, and $\Lambda_{+}\left(\hat{k}_{o n}\right)=\left(\hat{k}_{o n}+m\right) / 2 m$ is the positive energy spinor projector. The operator $g_{0}(K)$ is three-dimensional and depends on the kinematic variables $\left(\hat{k}_{i}^{+}, \hat{k}_{i \perp}\right)$ only. It is a global propagator, since it mediates propagation between hyperplanes according to Eq. (21), not allowing different lightfront times for each particle. It does not possess explicit covariance but is still covariant under light-front boosts. The global propagator $g_{0}(K)$ allows only physical particle propagation which has the plus component of the momentum positive, and contains only intermediate two-body states, with $K^{+}>0$. This is an advantage of using light-front dynamics. For example, in a system in which the lowest Fock-state component is composed of a particle and antiparticle, the individual physical plus momentum, as well as the total, are positive and in this case $g_{0}(K)$ propagates only particle and antiparticle intermediate two-body states.

The matrix element $\left\langle k_{1}^{\prime-}\left|\bar{G}_{0}(K)\right| k_{1}^{-}\right\rangle$of Eq. (22), in which only the dependence on $k_{1}^{-}$is explicit, is still an operator with respect to functions of the "kinematic" variables $\left(\hat{k}_{1}^{+}, \hat{\vec{k}}_{1 \perp}\right), \quad \hat{k}_{1 \text { on }}^{-}=\left(\hat{\vec{k}}_{1 \perp}^{2}+m_{1}^{2}\right) / \hat{k}_{1}^{+}$, and $\hat{k}_{2 o n}^{-}=\left[\left(\vec{K}_{\perp}-\hat{\vec{k}}_{1 \perp}\right)^{2}\right.$ $\left.+m_{2}^{2}\right] /\left(K^{+}-\hat{k}_{1}^{+}\right)$. The vertical bar indicates that the dependence on $k_{1}^{-}$is integrated out in Eq. (23). The bar on the left of the Green's function represents integration on $k_{1}^{-}$in the bra-state, the bar on the right in the ket state. The bar being placed on one side only of a Green's function represents the integration of $k_{1}^{-}$on that side alone.

The basis states for spinorial functions of the kinematical light-front variables are defined only for the positive energy sector of the spinor space by

$$
\left\langle x^{-} \vec{x}_{\perp} \mid k^{+} \vec{k}_{\perp} s\right\rangle=e^{-i\left(\frac{1}{2} k^{+} x^{-}-\vec{k}_{\perp} \cdot \vec{x}_{\perp}\right)} u(k, s),
$$

where the light-front spinor is

$$
u(k, s)=\frac{\left(k_{o n}+m\right)}{\sqrt{2 k^{+} 2 m}} \gamma^{+} \gamma^{0}\left(\begin{array}{c}
\chi_{s} \\
0
\end{array}\right),
$$

with $\chi_{s}$ being the two-component Pauli spinor. The lightfront spinors are normalized such that the positive-energy spinor projector is

$$
\Lambda_{+}\left(k_{o n}\right)=\sum_{s} u(k, s) \bar{u}(k, s) .
$$

The basis states are eigenfunctions of the momentum operators $\left(\hat{k}_{i}^{+}, \hat{k}_{i \perp}\right)$ and of the free energy operator $\hat{k}_{\text {ion }}^{-}$acting on functions of the kinematical variables. The states $\left|k^{+} \vec{k}_{\perp} s\right\rangle$ form a complete basis in the space of positive energy spinor functions of the kinematical variables, e.g.,

$$
\sum_{s} \int \frac{d k^{+} d^{2} k_{\perp}}{2(2 \pi)^{3}}\left|k^{+} \vec{k}_{\perp} s\right\rangle\left\langle k^{+} \vec{k}_{\perp} s\right| \gamma^{0}=\mathbf{1} .
$$

The auxiliary four-dimensional Green's function $\widetilde{G}_{0}(K)$ introduced in Eqs. (13)-(18) is defined, according to Ref. [8], such that the light-front propagators in higher Fock states appear explicitly in the kernel of integral equation for the auxiliary transition matrix, which will be given in the next section. It is written as

$$
\widetilde{G}_{0}(K):=\bar{G}_{0}(K)\left|g_{0}(K)^{-1}\right| \bar{G}_{0}(K) .
$$

The reduced Green's function $g_{0}(K)$ is defined only in the positive energy spinor subspace. It has an inverse there, with the inverse $g_{0}(K)^{-1}$ following from the definition in Eq. (25) as

$$
\begin{aligned}
g_{0}(K)^{-1}:= & -i \theta\left(K^{+}-\hat{k}_{1}^{+}\right) \theta\left(\hat{k}_{1}^{+}\right) \Lambda_{+}\left(\hat{k}_{1 o n}\right) \Lambda_{+}\left(\hat{k}_{2 o n}\right) \\
& \times \hat{k}_{1}^{+}\left(K^{+}-\hat{k}_{1}^{+}\right)\left(K^{-}-\hat{k}_{1 o n}^{-}-\hat{k}_{2 o n}^{-}+i o\right) .
\end{aligned}
$$

The auxiliary Green's function has the following useful properties:

$$
\begin{gathered}
\widetilde{G}_{0}(K)\left|=\bar{G}_{0}(K)\right|, \\
\left|\widetilde{G}_{0}(K)=\right| \bar{G}_{0}(K), \\
\left|\widetilde{G}_{0}(K)\right|=\left|\bar{G}_{0}(K)\right|,
\end{gathered}
$$

and defines a three-dimensional light-front transition matrix $t(K)$ through

$$
\begin{array}{r}
\left|\left[\widetilde{G}_{0}(K)+\widetilde{G}_{0}(K) T(K) \widetilde{G}_{0}(K)\right]\right| \\
\quad=g_{0}(K)+g_{0}(K) t(K) g_{0}(K) .
\end{array}
$$

We remind the reader that the bar signifies the integration of the $k_{1}^{-}$dependence of operators. Explicit matrix element of the auxiliary quantities are computed in Appendix A. 


\section{LIGHT-FRONT TRANSITION MATRIX AND BOUND-STATE WAVE FUNCTION}

Following Ref. [8], the four-dimensional transition matrix $T(K)$ can be obtained from the three-dimensional light-front one $t(K)$ defined by Eq. (35). The latter can be written as

$$
t(K)=g_{0}(K)^{-1}\left|\bar{G}_{0}(K) T(K) \bar{G}_{0}(K)\right| g_{0}(K)^{-1},
$$

by first iterating the integral equation, Eq. (13), once,

$$
T(K)=W(K)+W(K)\left[\widetilde{G}_{0}(K)+\widetilde{G}_{0}(K) T(K) \widetilde{G}_{0}(K)\right] W(K),
$$

and using the definition and properties of $\widetilde{G}_{0}(K)$, Eq. (30), and Eq. (36). The on-mass-shell matrix elements of $T(K)$, which define the two-fermion scattering amplitude are identical to the ones obtained from $t(K)$, as follows directly from Eq. (36) and the analytical properties of the $k^{-}$integration discussed in Appendix A. The scattering operator $T(K)$ is determined by $t(K)$ as

$$
\begin{aligned}
T(K)= & W(K)+W(K) \bar{G}_{0}(K) \\
& \times\left|\left[g_{0}(K)^{-1}+t(K)\right]\right| \bar{G}_{0}(K) W(K) .
\end{aligned}
$$

The light-front transition matrix $t(K)$ is the solution of the three-dimensional integral equation

$$
t(K)=w(K)+w(K) g_{0}(K) t(K),
$$

obtained from Eqs. (36) and (13), where the driving term $w(K)$ is obtained from the four-dimensional interaction $W(K)$ of Eq. (16) according to

$$
w(K):=g_{0}(K)^{-1}\left|\bar{G}_{0}(K) W(K) \bar{G}_{0}(K)\right| g_{0}(K)^{-1} .
$$

It is important to notice that the on- $k^{-}$-shell matrix elements of the light-front scattering amplitude, obtained from the perturbative solution of Eq. (38), match exactly the matrix elements of the perturbative covariant ladder scattering amplitude for on-mass-shell fermions at the same order in $g_{S}$. The reason is the equality expressed by Eq. (36) together with the effective interaction $w(K)$, from Eq. (39), calculated at the same perturbative order in $g_{S}$. We should also consider that in the evaluation of the matrix elements of Eq. (36) between states on the $k^{-}$shell, the inverse of the global propagator $g_{0}(K)$ cancels exactly the effect of the operator $\bar{G}_{0}(K)$, as discussed in Appendix A, and the right-side becomes equal to the matrix element of $T(K)$ between onmass-shell states.

If a bound-state pole of the transition matrix $T(K)$ exists at total four momentum $K_{B}, K_{B}^{2}=M_{B}^{2}$, it is also present in the three-dimensional transition matrix $t(K)$ at exactly the same $K_{B}$, due to Eq. (36). The vertex function $\left|\gamma_{B}\right\rangle$ of the bound-state is the solution of the homogeneous threedimensional equation

$$
\left|\gamma_{B}\right\rangle=w\left(K_{B}\right) g_{0}\left(K_{B}\right)\left|\gamma_{B}\right\rangle
$$

The four-dimensional vertex function $\left|\Gamma_{B}\right\rangle$ of the BSE at the bound-state pole can be obtained from $\left|\gamma_{B}\right\rangle$ by using Eq. (37),

$$
\left.\left|\Gamma_{B}\right\rangle=W\left(K_{B}\right) \bar{G}_{0}\left(K_{B}\right) \| \gamma_{B}\right\rangle \text {. }
$$

The BSE bound-state amplitude is found from the vertex function as

$$
\left.\left|\Psi_{B}\right\rangle=G_{0}^{F}\left(K_{B}\right) W\left(K_{B}\right) \bar{G}_{0}\left(K_{B}\right) \| \gamma_{B}\right\rangle
$$

The BSE bound-state vertex function $\left|\Gamma_{B}\right\rangle$ is related to the three-dimensional light-front wave function $\left|\phi_{B}\right\rangle$, defined by

$$
\left|\phi_{B}\right\rangle:=g_{0}\left(K_{B}\right)\left|\gamma_{B}\right\rangle
$$

and satisfying

$$
\left|\phi_{B}\right\rangle=g_{0}\left(K_{B}\right) w\left(K_{B}\right)\left|\phi_{B}\right\rangle,
$$

through the projection onto the hyperplane $x^{+}=0$

$$
\int d k_{1}^{-}\left\langle k_{1}^{-}\left|\bar{G}_{0}(K)\right| \Gamma_{B}\right\rangle=\left|\phi_{B}\right\rangle .
$$

This result follows immediately from the properties of the vertex and wave functions, Eqs. (40), (41), and (43). The auxiliary bound-state wave function $\left|\phi_{B}\right\rangle$ is the projection of $\bar{G}_{0}(K)\left|\Gamma_{B}\right\rangle$, to equal individual light-front times $x_{i}^{+}=x^{+}$, taken on the hyperplane $x^{+}=0$. The extraction of the instantantaneous terms of the fermion propagator allow the projection of the remaining part of the Bethe-Salpeter amplitude onto equal individual light-front times.

When predicting physical observables, we may either work directly with covariant operators, using $\left|\Psi_{B}\right\rangle$ and/or the transition matrix $T(K)$ of the $\mathrm{BSE}$, or we may derive effective operators suited to the context of the threedimensional light-front bound state $\left|\phi_{B}\right\rangle$ and/or the threedimensional light-front transition matrix $t(K)$. We have discussed, in the context of a bosonic system [8], an example of the utility of the three-dimensional light-front wave-function for determining the bound-state matrix element of the electroweak current $\mathcal{J}^{\mu}(Q)$ in the elastic process. We repeat the basic steps in the fermion case in Appendix B.

We note that the normalization condition of the BetheSalpeter amplitude $\left|\Psi_{B}\right\rangle$, Eq. (17), can be rewritten as the normalization condition of its three-dimensional light-front version $\left|\phi_{B}\right\rangle$. The three-dimensional normalization condition is found by inserting Eq. (B2) in Eq. (17) taking into account Eq. (43).

\section{SOLUTION OF THE BETHE-SALPETER EQUATION IN THE LADDER APPROXIMATION}

We discuss a possible calculational strategy for solving the BSE in the ladder approximation here. Within the spirit of this paper, we consider three-dimensional auxiliary quantities, i.e., of the bound-state vertex $\left|\gamma_{B}\right\rangle$ of Eq. (40) and the transition matrix $t(K)$ of Eq. (38). The steps from $\left|\gamma_{B}\right\rangle$ to the 


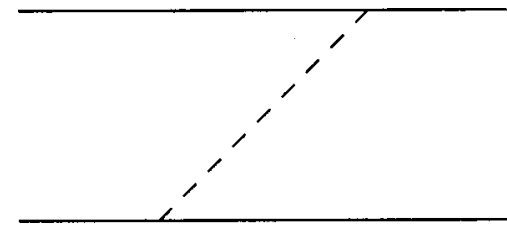

FIG. 1. Light-front time ordered diagram for $w^{(2)}(K)$ representing the light-front time ordered view of one $\sigma$ exchange.

four-dimensional BS bound-state vertex $\left|\Gamma_{B}\right\rangle$ and boundstate amplitude $\left|\Psi_{B}\right\rangle$ and from $t(K)$ to the four-dimensional transition matrix $T(K)$ should be carried out only when the full covariant results are required.
The auxiliary quantities $\left|\gamma_{B}\right\rangle$ and $t(K)$ are determined by the effective interaction $w(K)$, which has an expansion in powers of the coupling constant $g_{S}$,

$$
w(K)=\sum_{n=2}^{\infty} w^{(n)}(K) .
$$

It is hoped that this expansion converges rapidly. We therefore study its expansion up to order $g_{S}^{4}$,

$$
w(K)=w^{(2)}(K)+w^{(4)}(K) .
$$

Both contributions to Eq. (47) are given in terms of the driving term $V(K)$ of the BSE, using Eqs. (18) and (39), as

$$
\begin{aligned}
& w^{(2)}(K)=g_{0}(K)^{-1}\left|\bar{G}_{0}(K) V(K) \bar{G}_{0}(K)\right| g_{0}(K)^{-1}, \\
& w^{(4)}(K)=g_{0}(K)^{-1}\left|\bar{G}_{0}(K) V(K) G_{0}^{F}(K) V(K) \bar{G}_{0}(K)\right| g_{0}(K)^{-1}-g_{0}(K)^{-1}\left|\bar{G}_{0}(K) V(K) \widetilde{G}_{0}(K) V(K) \bar{G}_{0}(K)\right| g_{0}(K)^{-1} \\
& =g_{0}^{-1}(K)\left|\bar{G}_{0}(K) V(K)\left[\bar{G}_{0}(K)-\bar{G}_{0}(K)\left|g_{0}^{-1}(K)\right| \bar{G}_{0}(K)\right] V(K) \bar{G}_{0}(K) g_{0}^{-1}(K)\right| \\
& +g_{0}^{-1}(K)\left|\bar{G}_{0}(K) V(K) \Delta \bar{G}_{0}^{F}(K) V(K) \bar{G}_{0}(K)\right| g_{0}^{-1} .
\end{aligned}
$$

Equations (40) and (38) for the vertex $\left|\gamma_{B}\right\rangle$ and the transition matrix $t(K)$ are integral equations with the kernel $w(K) g_{0}(K)$. A common technique for solving the homogeneous equation (40) for $\left|\gamma_{B}\right\rangle$ or the inhomogeneous one (38) for $t(K)$ is to iterate that kernel. With the approximation (47) for the effective interaction $w(K)$ up to order $g_{S}^{4}$, the once-iterated kernel can be written and rearranged as

$$
\begin{aligned}
w(K) g_{0}(K)+\left[w(K) g_{0}(K)\right]^{2}= & \left\{w^{(2)}(K)+w^{(4)}(K)+\left[w^{(2)}(K)+w^{(4)}(K)\right] g_{0}(K)\left[w^{(2)}(K)+w^{(4)}(K)\right]\right\} g_{0}(K) \\
= & \left\{w^{(2)}(K)+\left[w^{(4)}(K)+w^{(2)}(K) g_{0}(K) w^{(2)}(K)\right]+\left[w^{(2)}(K) g_{0}(K) w^{(4)}(K)\right.\right. \\
& \left.\left.+w^{(4)}(K) g_{0}(K) w^{(2)}(K)\right]+w^{(4)}(K) g_{0}(K) w^{(4)}(K)\right\} g_{0}(K) .
\end{aligned}
$$

The discussion of this section will mainly be concerned with the contribution $\left[w^{(4)}(K)+w^{(2)}(K) g_{0}(K) w^{(2)}(K)\right]$ of order $g_{S}^{4}$. We will show that the two terms in this contribution have singularities which cancel. Thus the need for an artificial and ad hoc counterterm for $w^{(2)}(K) g_{0}(K) w^{(2)}(K)$, introduced in Ref. [5], does not exist. We demonstrate the cancellation of the two singularities next. In our opinion it is the most important observation of this section. However, we observe without further discussion that, in order to cancel the transverse singularities of $w^{(2)}(K) g_{0}(K) w^{(4)}(K)+w^{(4)}(K) g_{0}(K) w^{(2)}(K)$ $+w^{(4)}(K) g_{0}(K) w^{(4)}(K)$, the expansion of Eq. (50) should be performed up to $\left[w(K) g_{0}(K)\right]^{3}$ with the effective interaction expanded up to order $g_{S}^{6}$.

The operator $w^{(2)}(K)$ (see Fig. 1) is computed and discussed in Appendix C, the operator $w^{(4)}(K)$ in Appendix D. The operator $w^{(4)}(K)$ is not finite and it is divided in Appendix D into the two terms of Eq. $(49), w^{(4)}(K)=w_{\text {prop }}^{(4)}(K)$ $+w_{\text {inst }}^{(4)}(K)$. Due to the propagator difference of $\left[\bar{G}_{0}(K)-\bar{G}_{0}(K)\left|g_{0}(K)^{-1}\right| \bar{G}_{0}(K)\right]$ in $w_{\text {prop }}^{(4)}(K)$, the reducible fourth order term, $w^{(2)}(K) g_{0}(K) w^{(2)}(K)$, does not occur in $w^{(4)}(K)$. The second term of $w^{(4)}(K)$, i.e., $w_{\text {inst }}^{(4)}(K)$, contains the instantaneous part $\Delta \bar{G}_{0}^{F}$ of the free two-fermion propagator.

The three-dimensional quantities are written in the basis of light-front kinematic momenta of fermion $1\left(k_{1}^{+}, \vec{k}_{1 \perp}\right)$. All operators also depend on the total momentum $K$ for which $\vec{K}_{\perp}=0$ is chosen without any loss of generality. The mass of the interacting system is $M^{2}=K^{2}$ with $K^{+}>0$. It is then more convenient to use the basis $\left(x, \vec{k}_{1 \perp}\right)$ with $x:=k_{1}^{+} / K^{+}$for computations. For the sake of simplicity in the notation, the matrix element of the effective interaction is written as $w\left(y, \vec{k}_{1 \perp}^{\prime} ; x, \vec{k}_{1 \perp}\right)=\left\langle k_{1}^{\prime+} \vec{k}_{1 \perp}^{\prime}|w(K)| k_{1}^{+} \vec{k}_{1 \perp}\right\rangle$.

The perturbative "box counterterm," according to Ref. [5], is given in terms of the divergent part of the operator $w^{(I T E)}$ $=w^{(2)}(K) g_{0}(K) w^{(2)}(K)$, 


$$
w^{(I T E)}\left(y, \vec{k}_{1 \perp}^{\prime} ; x, \vec{k}_{1 \perp}\right)=-\frac{1}{(2 \pi)^{3}} \int \frac{d^{2} p_{1 \perp} d z \theta\left(\Lambda-\left|\vec{p}_{1 \perp}\right|\right)}{2 z(1-z)} \frac{w^{(2)}\left(y, \vec{k}_{1 \perp}^{\prime} ; z, \vec{p}_{1 \perp}\right) w^{(2)}\left(z, \vec{p}_{1 \perp} ; x, \vec{k}_{1 \perp}\right)}{M^{2}-\frac{\vec{p}_{1 \perp}^{2}+m^{2}}{z(1-z)}+i o} .
$$

The "box counterterm" $w^{(B C T)}$ is the $\Lambda \rightarrow \infty$ limit of $-w^{(I T E)}$, and takes the form

$$
\begin{aligned}
w^{(B C T)}\left(y, \vec{k}_{1 \perp}^{\prime} ; x, \vec{k}_{1 \perp}\right)= & -\frac{\left(g_{S}\right)^{4}}{8(2 \pi)^{2}\left(K^{+}\right)^{2}} \ln \Lambda \Lambda_{+}\left(k_{1 \text { on }}^{\prime}\right) \gamma_{1}^{+} \Lambda_{+}\left(k_{1 \text { on }}\right) \Lambda_{+}\left(\left(K-k_{1}^{\prime}\right)_{\text {on }}\right) \gamma_{2}^{+} \Lambda_{+}\left(\left(K-k_{1}\right)_{\text {on }}\right) \\
& \times\left\{\theta(x-y)\left[\frac{x-y}{(1-y) x}-\frac{1}{x}-\frac{\ln (1-y)}{x y}-\frac{1}{1-y}-\frac{\ln x}{(1-y)(1-x)}\right]+\theta(y-x)[x \leftrightarrow y]\right\} .
\end{aligned}
$$

Using that $\bar{u}\left(k^{\prime}, s^{\prime}\right) \gamma^{+} u(k, s)=\sqrt{k^{\prime+} k^{+}} / m$, the matrix element of the effective interaction can be written as

$$
\bar{u}\left(k_{1}^{\prime}, s_{1}^{\prime}\right) \bar{u}\left(k_{2}^{\prime}, s_{2}^{\prime}\right) w^{(B C T)}\left(y, \vec{k}_{1 \perp}^{\prime} ; x, \vec{k}_{1 \perp}\right) u\left(k_{1}, s_{1}\right) u\left(k_{2}, s_{2}\right) \equiv w^{(B C T)}(y ; x),
$$

since it does not depend on the spin projections and on the transverse momentum. It is given by

$$
\begin{aligned}
w^{(B C T)}(y ; x)= & -\frac{\left(g_{S}\right)^{4}}{8(2 \pi m)^{2}} \sqrt{y(1-y) x(1-x)} \\
& \times \ln \Lambda\left\{\theta(y-x)\left[\frac{y-x}{(1-x) y}-\frac{1}{y}-\frac{\ln (1-x)}{x y}-\frac{1}{1-x}-\frac{\ln y}{(1-x)(1-y)}\right]+\theta(x-y)[x \leftrightarrow y]\right\}
\end{aligned}
$$

In short, the divergent part of Eq. (53) can be compared to the "box counterterm" obtained in Ref. [5] and, up to a phase space factor and a factor of $i^{2}$, it agrees with the corresponding formula of that work.

The second order effective interaction, Eqs. (D12)-(D21), is derived in detail in Appendix D. The $d^{2} p_{1 \perp}$ integrations in the matrix elements of the effective interaction in order $g_{S}^{4}$ diverge logarithmicaly when the external momentum are kept fixed. They must be regularized and, to do this, we use a transversal momentum cutoff $\Lambda$. For $\Lambda \rightarrow \infty$, the sum of the effective interaction terms reproduce the counterterm presented in Ref. [5] and given by Eq. (53).

The contribution to the kernel from the interaction evaluated at order $g_{S}^{4}$ has two types of terms, $w^{(4)}=w_{\text {prop }}^{(4)}+w_{\text {inst }}^{(4)}$. The first term $w_{\text {prop }}^{(4)}$ comes from the propagating part of the Dirac propagators of Fig. 2(a), obtained from Eq. (D12) as detailed in Appendix D. It includes the virtual four-body propagation. The second type comes from the contribution of the instantaneous terms, $w_{\text {inst }, \alpha}^{(4)}(\alpha=1, \ldots, 8)$ obtained from Eqs. (D14)-(D21). The diagrams of Figs. 2(b)-2(d) represent schematically the contribution of the instantaneous term of the Dirac propagator to the effective interaction. The diagram in Fig. 2(b) represents the effective interaction for $\alpha=1-4$; Fig. 2(c) represents the effective interaction for $\alpha=5$ and 6; and Fig. 2(d) represents the effective interaction for $\alpha=7$ and 8. The effective interaction at order $\left(g_{S}\right)^{4}$ is

$$
w^{(4)}=w_{\text {prop }}^{(4)}+\sum_{\alpha=1}^{8} w_{i n s t, \alpha}^{(4)} .
$$

The matrix elements of the divergent part of each component of the effective interaction are given below. They do not depend on the spin projection nor on the transverse momentum, so that

$$
\bar{u}\left(k_{1}^{\prime}, s_{1}^{\prime}\right) \bar{u}\left(k_{2}^{\prime}, s_{2}^{\prime}\right) w_{\text {prop/inst }}^{(4)}\left(y, \vec{k}_{1 \perp}^{\prime} ; x, \vec{k}_{1 \perp}\right) u\left(k_{1}, s_{1}\right) u\left(k_{2}, s_{2}\right) \equiv w_{\text {prop/inst }}^{(4)}(y ; x) .
$$
is

The divergent part of the effective interaction at order $\left(g_{S}\right)^{4}$ due to the light-front four-body intermediate state propagation

$$
\begin{aligned}
w_{\text {prop }}^{(4)}(y ; x)= & \frac{g^{4}}{8(2 \pi m)^{2}} \sqrt{y(1-y) x(1-x)} \ln \Lambda \\
& \times\left\{\frac{\theta(x-y)}{(1-y)(x-y) x}\left[x-y-(1-y)(1-x) \ln \frac{(1-y)}{(1-x)}+x y \ln \frac{y}{x}\right]+\theta(y-x)[x \leftrightarrow y]\right\} .
\end{aligned}
$$

The divergent part of the effective interaction at order $\left(g_{S}\right)^{4}$ due to the instantaneous terms of the Dirac propagator is given as the sum of 


$$
\begin{aligned}
w_{\text {inst }, 1}^{(4)}(y ; x)+w_{\text {inst }, 2}^{(4)}(y ; x)= & -\frac{g^{4}}{8(2 \pi m)^{2}} \sqrt{y(1-y) x(1-x)} \ln \Lambda \\
& \times\left\{\frac{\theta(x-y)}{(1-y)(x-y)}\left[y \ln \frac{y}{x}+(1-y) \ln \frac{(1-y)}{(1-x)}\right]+\frac{\theta(y-x)}{y(y-x)}\left[y \ln \frac{y}{x}+(1-y) \ln \frac{(1-y)]}{(1-x)}\right]\right\}, \\
w_{\text {inst }, 3}^{(4)}(y ; x)+w_{\text {inst }, 4}^{(4)}(y ; x)= & -\frac{g^{4}}{8(2 \pi m)^{2}} \sqrt{y(1-y) x(1-x)} \ln \Lambda \\
& \times\left\{\frac{\theta(x-y)}{x(x-y)}\left[x \ln \frac{x}{y}+(1-x) \ln \frac{(1-x)}{(1-y)}\right]+\frac{\theta(y-x)}{(1-x)(y-x)}\left[(1-x) \ln \frac{1-x}{1-y}+x \ln \frac{x}{y}\right]\right\}, \\
w_{\text {inst }, 5}^{(4)}(y ; x)+w_{\text {inst }, 6}^{(4)}(y ; x)= & \frac{g^{4}}{8(2 \pi m)^{2}} \sqrt{y(1-y) x(1-x) \ln \Lambda} \\
& \times\left\{\frac{\theta(x-y)}{(1-x)(1-y)} \ln x+\frac{\theta(y-x)}{(1-x)(1-y)} \ln y+\frac{\theta(y-x)}{x y} \ln (1-x)+\frac{\theta(x-y)}{x y} \ln (1-y)\right\},
\end{aligned}
$$

and

$$
w_{\text {inst }, 7}^{(4)}(y ; x)+w_{\text {inst }, 8}^{(4)}(y ; x)=\frac{g^{4}}{8(2 \pi m)^{2}} \sqrt{y(1-y) x(1-x)} \ln \Lambda\left\{\frac{\theta(x-y)}{(x-y)} \ln \frac{x(1-y)}{y(1-x)}+\frac{\theta(y-x)}{(y-x)} \ln \frac{(1-x) y}{(1-y) x}\right\}
$$

Many cancellations occur in the sum of the divergent terms, Eqs. (55)-(59), of the effective interaction in order $\left(g_{S}\right)^{4}$. The final result is

$$
\begin{aligned}
w^{(4)}(y ; x)= & -\frac{\left(g_{S}\right)^{4}}{8(2 \pi m)^{2}} \sqrt{y(1-y) x(1-x)} \ln \Lambda \\
& \times\left\{\theta(y-x)\left[\frac{y-x}{(1-x) y}-\frac{1}{y}-\frac{\ln (1-x)}{x y}-\frac{1}{1-x}-\frac{\ln y}{(1-x)(1-y)}\right]+\theta(x-y)[x \leftrightarrow y]\right\},
\end{aligned}
$$

which exactly matches the result found for the "box counterterm,' $w^{(B C T)}(y ; x)$, in Eq. (53).

The three-dimensional integral equation for the vertex function $\left|\gamma_{B}\right\rangle$, with the effective interaction expanded up to fourth order in the coupling constant $g_{s}$, is developed in Appendix E. It is given by

$$
\left|\gamma_{B}\right\rangle=\left[w^{(2)}\left(K_{B}\right)+w^{(4)}\left(K_{B}\right)\right] g_{0}\left(K_{B}\right)\left|\gamma_{B}\right\rangle
$$

We remind the reader that, although the effective interaction $w^{(2)}\left(K_{B}\right)$ is well-behaved, the kernel $\left[w^{(2)}\left(K_{B}\right)\right] g_{0}\left(K_{B}\right)$ decreases only weakly like, $\left|k_{1 \perp}\right|^{-2}$ for large momenta. However, since some spin components of the vertex $\left|\gamma_{B}\right\rangle$ may become constant for large momenta, due to quite general considerations given in Ref. [5], converged solutions of Eq. (61), with only $w^{(2)}\left(K_{B}\right)$ cannot be obtained without regularizing the integral equation.

In a physically sound but mathematically nonconvergent theory, the suppression of large momenta by cutoffs is an acceptable brute-force method of avoiding singularities. Regularization by cutoffs is considered a rough approximation to the regularization of physics processes, too complex to be taken into account explicitly. The prediction of observ- ables depends on these cutoffs. The cutoffs are tuned to some of the observables, giving the regularized theory predicting power with respect to other observables. The need for regularization is different for the field-theoretic problem at hand, defined by the interaction Langrangian of Eq. (12). It is known that a four-dimensional bound state exists for the BSE in the ladder approximation, without any regularization by cutoff. If singularities occur in the steps chosen to obtain that four-dimensional bound state, the calculation procedure should be rearranged in order to avoid them completely or to balance them naturally.

The projection of the ladder Bethe-Salpeter equation on the light-front is performed systematically by the expansion in powers of $g_{S}$ of the reduced interaction $w(K)$ according to Eqs. (18) and (39). The kernel of the projected bound state equation for the vertex function, Eq. (40), can in principle be calculated to any order $n$ of $g_{S}$. The ladder light-front BetheSalpeter equation for the two-fermion bound-state in the Yukawa model, with the kernel including only intermediate three-particle states, must be renormalized [5]. We have shown above that the perturbative counterterms required to renormalize it, arises naturally in the systematic expansion of the interaction $w(K)$ given in Eq. (18). However, it is known 


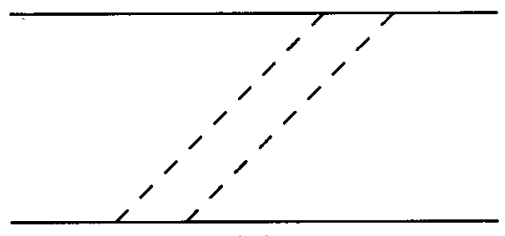

(a)

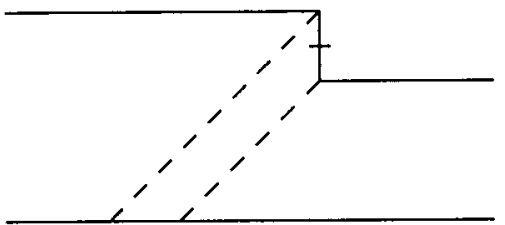

(b)

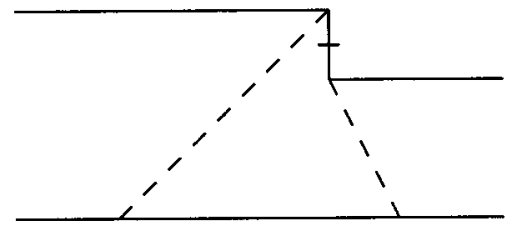

(c)

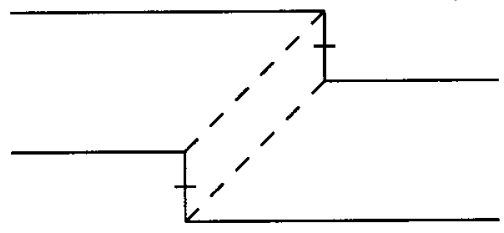

(d)

FIG. 2. Light-front time ordered diagram for $w^{(4)}(K)$ representing the light-front time ordered view of two $\sigma$ exchanges between two fermions. Diagram (a) represents $w_{\text {prop }}^{(4)}$ in which the simultaneous propagation of two $\sigma$ 's and two fermions occurs between the creation and annihilation of the bosons. Diagram (b) represents $w_{\text {inst }, \alpha}^{(4)}$ with $\alpha=1-4$, in which the contribution of the instantaneous term of the Dirac propagator of one of the fermions appears (represented by the vertical line). Diagram (c) represents $w_{\text {inst }, \alpha}^{(4)}$ with $\alpha=5,6$, in which the instantaneous term of one of the fermion propagators again appears. Diagram (d) represents $w_{i n s t, \alpha}^{(4)}$ with $\alpha$ $=7,8$, in which both intermediate fermions have instantaneous propagation.

that the perturbative "box counterterm" decreases the sensitivity to the transverse momentum cutoff, but it does not renormalize the theory and permit the prediction of the mass of the lowest bound state [5] without further renormalization, even though the four-dimensional ladder Bethe-Salpeter has a well-defined ground state mass for the bound two-fermion system.

\section{CONCLUSION}

This work generalizes to the fermionic case a calculational procedure applied for solving the light-front BSE for bosons. This procedure uses a three-dimensional integral equation, in the framework of light-front dynamics, to produce the solution to the BSE by quadrature. The intermediate three-dimensional quantities are only covariant under kinematical light-front boosts. Full covariance is restored in the final step to the solution to the BSE.

The formalism is exact, offering an efficient approximative scheme in which only intermediate two-fermion states propagate. All the complexity of many-body and antiparticle propagation is contained in the effective three-dimensional interaction. At the level of the ladder approximation the antiparticle propagation is included in the instantaneous term of the Dirac propagator which is cointained in the effective interaction. Systematical improvement of the calculation is possible through the expansion of the effective interaction to higher orders of the coupling constant.

The systematic expansion of the effective interaction shows in principle how to handle the renormalization problem of the ladder BS equation, as exemplified in the Yukawa model, at order $\left(g_{S}\right)^{4}$. The so-called "box counterterm" for renormalizing $w^{(2)}(K) g_{0}(K) w^{(2)}(K)$ appears naturally in the expansion of the effective interaction. It is the sum of ten diagrams in which cancellations between different terms result in the proposed counterterm. At each order of the systematic expansion of the kernel of the ladder equation, the cutoff is still required although sensitivity to the cutoff should decrease as we proceed in the expansion. However, the four-dimensional ladder BS equation in the Yukawa model need not be renormalized. It is natural that the counterterms are generated by the expansion, as we are approaching the true solution of the BS equation. The cancellation of singularities occurs at all orders in the expansion of the kernel of the light-front BS equation, because the expansion of the kernel together with the iteration of the light-front $T$-matrix equation necessarily reproduces the perturbative covariant ladder scattering amplitude at the given power in $g_{S}$. At least in principle, at the ladder level, we have shown that the light-front theory does not need any counterterms beside those already included in the expansion of the effective interaction.

A last comment is appropriate here. The computation time required to solve the homogeneous light-front BSE for a two-boson bound state with a kernel calculated at order $g_{S}^{4}$, by means of discretization through Gaussian-Legendre quadrature and iteration [8], is quite modest, in a present day worskstation. The computation time needed to solve the bound state problem in the Yukawa model with the above method, at order $g_{S}^{4}$, increases by about two orders of magnitude. To estimate it, we consider that the dimension of the matrix increases by a factor of 4 and the number of independent terms in the kernel is 5, Eqs. (E3), (E5)-(E8). We believe that the numerical solution of the bound state problem with the present formalism is within reach, but we leave it for a future work.

\section{ACKNOWLEDGMENTS}

TThe authors thank G.A. Miller for suggestions on the manuscript. The authors acknowledge partial support from the CAPES/DAAD/Probral Project No. 015/95. Part of this work was performed during the visits of T.F. and P.U.S. They thank the hospitality of the respective host institutions. 
Furthermore, the work was supported by a Brazilian grant (J.H.O.S.) from FAPESP and by research grants (B.V.C. and T.F.) from $\mathrm{CNPq}$ and FAPESP.

\section{APPENDIX A: EVALUATION OF AUXILIARY QUANTITIES}

The connection between the three-dimensional and four-dimensional equations is made through the operators $\bar{G}_{0}(K) \mid g_{0}(K)^{-1}$ and $g_{0}(K)^{-1} \mid \bar{G}_{0}(K)$. To discuss the dimensional reduction, we choose $\bar{G}_{0}(K) \mid g_{0}(K)^{-1}$. The momentum space matrix elements of $\bar{G}_{0}(K) \mid g_{0}(K)^{-1}$ for $K^{+}>0$, are

$$
\begin{aligned}
\left\langle k_{1}^{\prime-} k_{1}^{\prime+} \vec{k}_{1 \perp}^{\prime}\left|\bar{G}_{0}(K)\right| g_{0}(K)^{-1} \mid k_{1}^{+} \vec{k}_{1 \perp}\right\rangle= & \frac{i}{2 \pi} \delta\left(k_{1}^{\prime+}-k_{1}^{+}\right) \delta\left(\vec{k}_{1 \perp}^{\prime}-\vec{k}_{1 \perp}\right) \theta\left(K^{+}-k_{1}^{+}\right) \theta\left(k_{1}^{+}\right) \\
& \times \frac{\Lambda_{+}\left(k_{1 o n}^{\prime}\right)}{\left(k_{1}^{\prime-}-\frac{\vec{k}_{1 \perp}^{\prime 2}+m_{1}^{2}-i o}{k_{1}^{\prime+}}\right)} \frac{\Lambda_{+}\left(\left(K-k_{1}^{\prime}\right)_{o n}\right)}{\left(K^{-}-k_{1}^{\prime-}-\frac{\left(\vec{K}_{\perp}-\vec{k}_{1 \perp}^{\prime}\right)^{2}+m_{2}^{2}-i o}{K^{+}-k_{1}^{\prime+}}\right)} \\
& \times\left(K^{-}-k_{1 o n}^{-}-k_{2 o n}^{-}+i o\right) .
\end{aligned}
$$

If the light-front "energy" $K^{-}$is not on-shell, i.e., $K^{-} \neq k_{1 o n}^{-}+k_{2 o n}^{-}$, the evaluation of the matrix element in Eq. (A1) obtained from the integration on $k_{1}^{\prime-}$ can be carried out with usual techniques.

If the avalable light-front "energy" $K^{-}$is on-shell, i.e., $K^{-}=K_{o n}^{-}=k_{1 \text { on }}^{-}+k_{2 \text { on }}^{-}$the integration of $k^{\prime}{ }_{1}^{-}$should be performed with care using the concepts of distributions. In this case the matrix element will always be integrated with respect to $k^{\prime}-{ }_{1}$, but over a function $f\left(k^{\prime}{ }_{1}^{-}\right)$still to be determined and, unfortunately, with unknown analyticity properties. We will assume that $K^{+}>0$ and $k_{1}^{+}>0$, without a loss the generality. Thus, we have

$$
\begin{aligned}
& \int d k_{1}^{\prime-} f\left(k_{1}^{\prime-}\right)\left\langle k_{1}^{\prime-} k_{1}^{\prime+} \vec{k}_{1 \perp}^{\prime}\left|\bar{G}_{0}(K)\right| g_{0}(K)^{-1} \mid k_{1}^{+} \vec{k}_{1 \perp}\right\rangle \\
& \quad=\frac{i}{2 \pi} \delta\left(k_{1}^{\prime+}-k_{1}^{+}\right) \delta\left(\vec{k}_{1 \perp}^{\prime}-\vec{k}_{1 \perp}\right) \int d k_{1}^{\prime-} f\left(k_{1}^{\prime-}\right) \frac{\Lambda_{+}\left(k_{1 o n}\right)}{\left(k_{1}^{\prime-}-k_{1 o n}^{-}+i o\right)} \frac{\Lambda_{+}\left(k_{2 o n}\right)}{\left(K^{-}-k_{1}^{\prime-}-k_{2 o n}^{-}+i o\right)}\left(K^{-}-k_{1 o n}^{-}-k_{2 o n}^{-}+i o\right) .
\end{aligned}
$$

In general, $f\left(k_{1}^{\prime-}\right)$ can be split into a part $f_{u h p}\left(k_{1}^{\prime-}\right)$ having singularities only in the upper half ${k^{\prime}}_{1}^{-}$-plane and a part $f_{l h p}\left(k^{\prime-}\right)$ having singularities only in the lower half $k_{1}^{\prime-}$-plane,

$$
f\left(k_{1}^{\prime-}\right)=f_{u h p}\left(k_{1}^{\prime-}\right)+f_{l h p}\left(k_{1}^{\prime-}\right) .
$$

In the case that there are poles in both half planes, they can be fully separated,

$$
g\left(k_{1}^{\prime-}\right) \frac{1}{k_{1}^{\prime-}-\alpha_{1}-i \alpha_{2}} \frac{1}{k_{1}^{\prime-}-\beta_{1}+i \beta_{2}}=g\left(k_{1}^{\prime-}\right) \frac{1}{(\alpha-\beta)+i\left(\alpha_{2}+\beta_{2}\right)}\left[\frac{1}{k_{1}^{\prime-}-\alpha_{1}-i \alpha_{2}}-\frac{1}{k_{1}^{\prime-}-\beta_{1}+i \beta_{2}}\right] \text {, }
$$

with $g\left(k_{1}^{\prime}\right)$ being singularity free. The integration in Eq. (A2) can now be carried out using Cauchy's theorem:

$$
\begin{aligned}
\int d k_{1}^{-} f\left(k_{1}^{\prime-}\right)\left\langle k_{1}^{\prime-} k_{1}^{\prime+} \vec{k}_{1 \perp}^{\prime}\left|\bar{G}_{0}(K)\right| g_{0}(K)^{-1} \mid k_{1}^{+} \vec{k}_{1 \perp}\right\rangle \\
=\delta\left(k_{1}^{\prime+}-k_{1}^{+}\right) \delta\left(\vec{k}_{1 \perp}^{\prime}-\vec{k}_{1 \perp}\right)\left(K^{-}-k_{1 o n}^{-}-k_{2 o n}^{-}+i o\right) \\
\quad \times\left[f_{u h p}\left(k_{1 o n}^{-}\right) \frac{\Lambda_{+}\left(k_{1 o n}\right) \Lambda_{+}\left(k_{2 o n}\right)}{K^{-}-k_{1 o n}^{-}-k_{2 o n}^{-}+i o}+f_{l h p}\left(K^{-}-k_{2 o n}^{-}\right) \frac{\Lambda_{+}\left(k_{1 o n}\right) \Lambda_{+}\left(k_{2 o n}\right)}{K^{-}-k_{2 o n}^{-}-k_{1 o n}^{-}+i o}\right] \\
=\delta\left(k_{1}^{\prime+}-k_{1}^{+}\right) \delta\left(\vec{k}_{1 \perp}^{\prime}-\vec{k}_{1 \perp}\right)\left[f_{u h p}\left(k_{1 o n}^{-}\right)+f_{l h p}\left(K^{-}-k_{2 o n}^{-}\right)\right] \Lambda_{+}\left(k_{1 o n}\right) \Lambda_{+}\left(k_{2 o n}\right) .
\end{aligned}
$$

We note that the propagators cancel and no singularity remains. The importance of this result appears when the light-front "energy" is on-shell, $K^{-}=K_{o n}^{-}$. Then the two terms can be recombined to obtain the original function, i.e., 


$$
\int d k_{1}^{\prime-} f\left(k_{1}^{\prime-}\right)\left\langle k_{1}^{\prime-} k_{1}^{\prime+} \vec{k}_{1 \perp}^{\prime}\left|\bar{G}_{0}(K)\right| g_{0}(K)^{-1} \mid k_{1}^{+} \vec{k}_{1 \perp}\right\rangle=\delta\left(k_{1}^{\prime+}-k_{1}^{+}\right) \delta\left(\vec{k}_{1 \perp}^{\prime}-\vec{k}_{1 \perp}\right) f\left(k_{1 o n}^{-}\right) \Lambda_{+}\left(k_{1 o n}\right) \Lambda_{+}\left(k_{2 o n}\right)
$$

for $K^{-}=K_{o n}^{-}$.

\section{APPENDIX B: ELECTROWEAK CURRENT IN THE ELASTIC PROCESS}

The electroweak current $\mathcal{J}^{\mu}(Q)$ in the elastic process serves as an example of how to derive expressions for one particular observable using three-dimensional light-front operators and wave function. The current operator connects an initial bound state defined from the Bethe-Salpeter amplitude $\left|\Psi_{B i}\right\rangle$ to a final one $\left|\Psi_{B f}\right\rangle$ through an elastic process. The current operator, $\mathcal{J}^{\mu}(Q)$, is appropriately defined in field theory with a four-momentum transfer $Q=K_{B f}-K_{B i}$. The matrix element for describing the process $\left\langle\Psi_{B f}\left|\mathcal{J}^{\mu}(Q)\right| \Psi_{B i}\right\rangle$ can be derived from the Bethe-Salpeter bound-state amplitude $\left|\Psi_{B}\right\rangle$ as well as from the three-dimensional light-front bound state $\left|\phi_{B}\right\rangle$ through the relation

$$
\left\langle\Psi_{B f}\left|\mathcal{J}^{\mu}\left(K_{B f}-K_{B i}\right)\right| \Psi_{B i}\right\rangle=\left\langle\phi_{B f}\left|j^{\mu}\left(K_{B f}, K_{B i}\right)\right| \phi_{B i}\right\rangle
$$

Using the condition of Eq. (40), $\left|\gamma_{B}\right\rangle-w\left(K_{B}\right) g_{0}\left(K_{B}\right)\left|\gamma_{B}\right\rangle=0$, and the definition of $w(K)$, Eq. (39) in Eq. (42) the bound-state amplitude can be written in terms of the three-dimensional vertex function as

$$
\left.\left|\Psi_{B}\right\rangle=\left[1+\left(G_{0}^{F}\left(K_{B}\right)-\bar{G}_{0}\left(K_{B}\right)\left|g_{0}\left(K_{B}\right)^{-1}\right| \bar{G}_{0}\left(K_{B}\right)\right) W\left(K_{B}\right)\right] \bar{G}_{0}\left(K_{B}\right)|| \gamma_{B}\right\rangle .
$$

The effective current in three-dimensional space, which is deduced by introducing the $\left|\Psi_{B}\right\rangle$ given by Eq. (B2) and $\left|\phi_{B}\right\rangle$ from Eq. (43), separates in the kinematic and interaction-dependent parts Eq. (B1), as

$$
\begin{gathered}
j^{\mu}\left(K_{f}, K_{i}\right):=g_{0}\left(K_{f}\right)^{-1} \mid \bar{G}_{0}\left(K_{f}\right)\left[1+W\left(K_{f}\right)\left(G_{0}^{F}\left(K_{f}\right)-\bar{G}_{0}\left(K_{f}\right)\left|g_{0}\left(K_{f}\right)^{-1}\right| \bar{G}_{0}\left(K_{f}\right)\right)\right] \\
\mathcal{J}^{\mu}\left(K_{f}-K_{i}\right)\left[1+\left(G_{0}^{F}\left(K_{i}\right)-\bar{G}_{0}\left(K_{i}\right)\left|g_{0}\left(K_{i}\right)^{-1}\right| \bar{G}_{0}\left(K_{i}\right)\right) W\left(K_{i}\right)\right] \bar{G}_{0}\left(K_{i}\right) \mid g_{0}\left(K_{i}\right)^{-1} .
\end{gathered}
$$

The bound state has to be calculated for the initial and final four momenta $K_{B i}$ and $K_{B f}$. The effective current $j^{\mu}\left(K_{f}, K_{i}\right)$ is predominantly obtained kinematically from the covariant one as $g_{0}\left(K_{f}\right)^{-1}\left|\bar{G}_{0}\left(K_{f}\right) \mathcal{J}^{\mu}\left(K_{f}-K_{i}\right) \bar{G}_{0}\left(K_{i}\right)\right| g_{0}\left(K_{i}\right)^{-1}$, but it also depends on the interaction $W(K)$ of Eq. (16). If $W(K)$ is computed up to a certain order in the original interaction $V(K)$ of the BSE, the effective current should be expanded consistently up that order.

\section{APPENDIX C: INTERACTION IN FIRST ORDER}

The interaction $w(k)$, defined by Eqs. (39) and (16), to lowest order in the driving term $V(K)$, is given by

$$
w^{(2)}(K)=g_{0}(K)^{-1}\left|\bar{G}_{0}(K) V(K) \bar{G}_{0}(K)\right| g_{0}(K)^{-1},
$$

where the matrix element of the operator $\left|G_{0}(K) V(K) G_{0}(K)\right|$ is

$$
\begin{aligned}
\left\langle k_{1}^{\prime+} \vec{k}_{1 \perp}^{\prime}|| \bar{G}_{0}(K) V(K) \bar{G}_{0}(K)|| k_{1}^{+} \vec{k}_{1 \perp}\right\rangle & =i \frac{\left(4 m_{1} m_{2}\right)^{2}\left(i g_{S}\right)^{2}}{(2 \pi)^{2}} \int d k_{1}^{\prime-} d k_{1}^{-} \frac{1}{k_{1}^{\prime+}\left(K^{+}-k_{1}^{\prime+}\right)} \frac{\Lambda_{+}\left(k_{1 o n}^{\prime}\right)}{\left(k_{1}^{\prime-}-\frac{\vec{k}_{1 \perp}^{\prime 2}+m_{1}^{2}-i o}{k_{1}^{\prime+}}\right)} \\
& \times \frac{\Lambda_{+}\left(k_{2 o n}^{\prime}\right)}{\left(K^{-}-k_{1}^{\prime-}-\frac{\left(\vec{K}_{\perp}-\vec{k}_{1 \perp}^{\prime}\right)^{2}+m_{2}^{2}-i o}{K^{+}-k_{1}^{\prime+}}\right)} \frac{1}{\left(k_{1}^{\prime+}-k_{1}^{+}\right)} \frac{1}{\left(k_{1}^{\prime-}-k_{1}^{-}-\frac{\left(\vec{k}_{1}^{\prime}-\vec{k}_{1 \perp}\right)^{2}+\mu^{2}-i o}{k_{1}^{\prime+}-k_{1}^{+}}\right)} \\
& \left.\times \frac{\Lambda_{+}\left(k_{1 o n}\right)}{k_{1}^{+}\left(K^{+}-k_{1}^{+}\right)} \frac{\Lambda_{+}\left(k_{2 o n}\right)}{\left(k_{1}^{-}-\frac{\vec{k}_{1 \perp}^{2}+m_{1}^{2}-i o}{k_{1}^{+}}\right)} \frac{\left(\vec{K}_{\perp}-\vec{k}_{1 \perp}\right)^{2}+m_{2}^{2}-i o}{K^{+}-k_{1}^{+}}\right)
\end{aligned}
$$

The double integration in $k^{-}$in Eq. (C2) is performed analytically using Cauchy's theorem and the condition $K^{+}>0$. The integration is nonzero for $K^{+}>k_{1}^{\prime+}>0$ and $K^{+}>k_{1}^{+}>0$. Two possibilities also appear for $\sigma$ forward propagation. For $k_{1}^{+}$ $>k_{1}^{\prime+}$, a $\sigma$ is emitted by particle 1 . Otherwise, it is absorbed, so that 


$$
\begin{aligned}
\left\langle k_{1}^{\prime+} \vec{k}_{1 \perp}^{\prime}\right. & \left.\left\|\bar{G}_{0}(K) V(K) \bar{G}_{0}(K)\right\| k_{1}^{+} \vec{k}_{1 \perp}\right\rangle \\
= & \left(4 m_{1} m_{2}\right)^{2}\left(i g_{S}\right)^{2} \frac{\theta\left(K^{+}-k_{1}^{\prime+}\right) \theta\left(k_{1}^{\prime+}\right)}{k_{1}^{\prime+}\left(K^{+}-k_{1}^{+}\right)} \frac{i \Lambda_{+}\left(k_{1 o n}^{\prime}\right) \Lambda_{+}\left(k_{2 o n}^{\prime}\right)}{\left(K^{-}-k_{1 o n}^{\prime}-k_{2 o n}^{\prime-}+i o\right)} \\
& \times\left(\frac{\theta\left(k_{1}^{+}-k_{1}^{\prime+}\right)}{\left(k_{1}^{+}-k_{1}^{\prime+}\right)} \frac{i \Lambda_{+}\left(k_{1 o n}^{\prime}\right) \Lambda_{+}\left(k_{2 o n}\right)}{\left(K^{-}-k_{1 o n}^{\prime}-k_{2 o n}^{-}-k_{\sigma o n}^{\prime}+i o\right)}+\frac{\theta\left(k_{1}^{\prime+}-k_{1}^{+}\right)}{\left(k_{1}^{\prime+}-k_{1}^{+}\right)} \frac{i \Lambda_{+}\left(k_{1 o n}\right) \Lambda_{+}\left(k_{2 o n}^{\prime}\right)}{\left(K^{-}-k_{1 o n}^{-}-k_{2 o n}^{\prime-}-k_{\sigma o n}^{-}+i o\right)}\right) \\
& \times \frac{\theta\left(K^{+}-k_{1}^{+}\right) \theta\left(k_{1}^{+}\right)}{k_{1}^{+}\left(K^{+}-k_{1}^{+}\right)} \frac{i \Lambda_{+}\left(k_{1 o n}\right) \Lambda_{+}\left(k_{2 o n}\right)}{\left(K^{-}-k_{1 o n}^{-}-k_{2 o n}^{-}+i o\right)},
\end{aligned}
$$

where the light-front "energies" of the intermediate states of the individual particles are given by

$$
\begin{gathered}
k_{1 \text { on }}^{\prime-}=\frac{\vec{k}_{1 \perp}^{\prime 2}+m_{1}^{2}}{k_{1}^{\prime+}}, \\
k_{1 \text { on }}^{-}=\frac{\vec{k}_{1 \perp}^{2}+m_{1}^{2}}{k_{1}^{+}}, \\
k_{2 o n}^{\prime-}=\frac{\left(\vec{K}_{\perp}-\vec{k}_{1 \perp}^{\prime}\right)^{2}+m_{2}^{2}}{K^{+}-k_{1}^{\prime+}}, \\
k_{2 o n}^{-}=\frac{\left(\vec{K}_{\perp}-\vec{k}_{1 \perp}\right)^{2}+m_{2}^{2}}{K^{+}-k_{1}^{+}}, \\
k_{\text {\%on }}^{\prime-}=\frac{\left(\vec{k}_{1 \perp}^{\prime}-\vec{k}_{1 \perp}\right)^{2}+\mu^{2}}{k_{1}^{+}-k_{1}^{\prime+}}, \\
k_{\text {\%on }}^{-}=\frac{\left(\vec{k}_{1 \perp}^{\prime}-\vec{k}_{1 \perp}\right)^{2}+\mu^{2}}{k_{1}^{\prime+}-k_{1}^{+}} .
\end{gathered}
$$

The global three-particle propagator for 1, 2, and $\sigma$ appears in Eq. (C3), in two cases: when $\sigma$ is either emitted or absorbed by particle 1 .

The matrix element $\left\langle k^{\prime}{ }_{1}^{+} \vec{k}_{1 \perp}^{\prime}\left|w^{(2)}(K)\right| k_{1}^{+} \vec{k}_{1 \perp}\right\rangle$ is obtained from Eq. (C3) by multiplying both sides by the matrix element of the operator $g_{0}(K)^{-1}$, given in Eq. (24),

$$
\begin{aligned}
\left\langle k_{1}^{\prime+} \vec{k}_{1 \perp}^{\prime}\left|w^{(2)}(K)\right| k_{1}^{+} \vec{k}_{1 \perp}\right\rangle= & \left(i g_{S}\right)^{2} \frac{\theta\left(k_{1}^{+}-k_{1}^{\prime+}\right)}{\left(k_{1}^{+}-k_{1}^{\prime+}\right)} \frac{i \Lambda_{+}\left(k_{1 o n}^{\prime}\right) \Lambda_{+}\left(k_{1 o n}\right) \Lambda_{+}\left(k_{2 o n}^{\prime}\right) \Lambda_{+}\left(k_{2 o n}\right)}{\left(K^{-}-k_{1 o n}^{\prime}-k_{2 o n}^{-}-k_{\sigma o n}^{\prime}+i o\right)} \\
& +\left(i g_{S}\right)^{2} \frac{\theta\left(k_{1}^{\prime+}-k_{1}^{+}\right)}{\left(k_{1}^{\prime+}-k_{1}^{+}\right)} \frac{i \Lambda_{+}\left(k_{1 o n}^{\prime}\right) \Lambda_{+}\left(k_{1 o n}\right) \Lambda_{+}\left(k_{2 o n}^{\prime}\right) \Lambda_{+}\left(k_{2 o n}\right)}{\left(K^{-}-k_{1 o n}^{-}-k_{2 o n}^{\prime}-k_{\sigma o n}^{-}+i o\right)} \\
= & \left(i g_{S}\right)^{2} \frac{\theta\left(k_{1}^{+}-k_{1}^{\prime+}\right)}{\left(k_{1}^{+}-k_{1}^{\prime+}\right)} \frac{i \Lambda_{+}\left(k_{1 o n}^{\prime}\right) \Lambda_{+}\left(k_{1 o n}\right) \Lambda_{+}\left(k_{2 o n}^{\prime}\right) \Lambda_{+}\left(k_{2 o n}\right)}{\left(K^{-}-\frac{\vec{k}_{1 \perp}^{\prime 2}+m_{1}^{2}}{k_{1}^{\prime+}}-\frac{\left(\vec{K}_{\perp}-\vec{k}_{1 \perp}\right)^{2}+m_{2}^{2}}{K^{+}-k_{1}^{+}}-\frac{\left(\vec{k}_{1 \perp}^{\prime}-\vec{k}_{1 \perp}\right)^{2}+\mu^{2}}{k_{1}^{+}-k_{1}^{\prime+}}+i o\right)} \\
& +\left(i g_{S}\right)^{2} \frac{\theta\left(k_{1}^{\prime+}-k_{1}^{+}\right)}{\left(k_{1}^{\prime+}-k_{1}^{+}\right)} \frac{i_{+}\left(k_{1 o n}^{\prime}\right) \Lambda_{+}\left(k_{1 o n}\right) \Lambda_{+}\left(k_{2 o n}^{\prime}\right) \Lambda_{+}\left(k_{2 o n}\right)}{\left(K^{-}-\frac{\vec{k}_{1 \perp}^{2}+m_{1}^{2}}{k_{1}^{+}}-\frac{\left(\vec{K}_{\perp}-\vec{k}_{1 \perp}^{\prime}\right)^{2}+m_{2}^{2}}{K^{+}-k_{1}^{\prime+}}-\frac{\left(\vec{k}_{1 \perp}^{\prime}-\vec{k}_{1 \perp}\right)^{2}+\mu^{2}}{k_{1}^{\prime+}-k_{1}^{+}}+i o\right)} .
\end{aligned}
$$


Above, the convention was used that the positive energy spinor projectors with argument of the type $(K-k)_{\text {on }}$ refer to the fermion labeled 2, while argument of the type $k_{\text {on }}$ refers to the fermion labeled 1.

\section{APPENDIX D: INTERACTION IN SECOND ORDER}

The interaction $w(k)$, defined by Eqs. (39) and (16) to second order in the driving term $V(K)$, is given by

$$
w(K) \simeq w^{(2)}(K)+w^{(4)}(K),
$$

where $w^{(2)}(K)$ is given by Eq. (C5) and

$$
w^{(4)}(K)=g_{0}(K)^{-1}\left|\bar{G}_{0}(K) V(K) G_{0}^{F}(K) V(K) \bar{G}_{0}(K)\right| g_{0}(K)^{-1}-g_{0}(K)^{-1}\left|\bar{G}_{0}(K) V(K) \widetilde{G}_{0}(K) V(K) \bar{G}_{0}(K)\right| g_{0}(K)^{-1}
$$

The second term in Eq. (D2) corresponds to the iteration of the interaction $w^{(2)}(K)$

$$
\begin{aligned}
g_{0}(K)^{-1}\left|\bar{G}_{0}(K) V(K) \widetilde{G}_{0}(K) V(K) \bar{G}_{0}(K)\right| g_{0}(K)^{-1} & =g_{0}(K)^{-1}\left|\bar{G}_{0}(K) V(K) \bar{G}_{0}(K)\right| g_{0}(K)^{-1}\left|\bar{G}_{0}(K) V(K) \bar{G}_{0}(K)\right| g_{0}(K)^{-1} \\
& =w^{(2)} g_{0}(K) w^{(2)}
\end{aligned}
$$

The matrix element of the operator $\left|\bar{G}_{0}(K) V(K) G_{0}^{F}(K) V(K) \bar{G}_{0}(K)\right|$ has two parts, one being $\left|\bar{G}_{0}(K) V(K) \bar{G}_{0}(K) V(K) \bar{G}_{0}(K)\right|$ and the other being the instantaneous term of the Dirac propagator, $\left|\bar{G}_{0}(K) V(K) \Delta G_{0}^{F}(K) V(K) \bar{G}_{0}(K)\right|$. We split the interaction in second order as $w^{(4)}=w_{\text {prop }}^{(4)}+w_{\text {inst }}^{(4)}$, where $w_{\text {prop }}^{(4)}$ contains the propagating part of the fermion propagator and $w_{i n s t}^{(4)}$ contains the instantaneous pieces. We begin the evaluation of $w_{p r o p}^{(4)}$ by calculating

$$
\begin{aligned}
& \left\langle k_{1}^{\prime+} \vec{k}_{1 \perp}^{\prime}\left\|\bar{G}_{0}(K) V(K) \bar{G}_{0}(K) V(K) \bar{G}_{0}(K)\right\| k_{1}^{+} \vec{k}_{1 \perp}\right\rangle \\
& =\frac{\left(4 m_{1} m_{2}\right)^{3}\left(i g_{S}\right)^{4}}{2(2 \pi)^{6}} \int d k_{1}^{\prime-} d p_{1}^{-} d k_{1}^{-} d p_{1}^{+} d^{2} p_{1 \perp} \frac{1}{k_{1}^{\prime+}\left(K^{+}-k_{1}^{\prime+}\right)} \frac{\Lambda_{+}\left(k_{1 \text { on }}^{\prime}\right)}{\left(k_{1}^{\prime-}-\frac{\vec{k}_{1 \perp}^{\prime}+m_{1}^{2}-i o}{k_{1}^{\prime+}}\right)} \\
& \times \frac{\Lambda_{+}\left(k_{2 o n}^{\prime}\right)}{\left(K^{-}-k_{1}^{\prime-}-\frac{\left(\vec{K}_{\perp}-\vec{k}_{1 \perp}^{\prime}\right)^{2}+m_{2}^{2}-i o}{K^{+}-k_{1}^{\prime+}}\right)} \frac{1}{\left(k_{1}^{\prime+}-p_{1}^{+}\right)} \frac{1}{\left(k_{1}^{\prime-}-p_{1}^{-}-\frac{\left(\vec{k}_{1 \perp}^{\prime}-\vec{p}_{1 \perp}\right)^{2}+\mu^{2}-i o}{k_{1}^{\prime+}-p_{1}^{+}}\right)} \\
& \times \frac{1}{p_{1}^{+}\left(K^{+}-p_{1}^{+}\right)} \frac{\Lambda_{+}\left(p_{1 o n}\right)}{\left(p_{1}^{-}-\frac{\vec{p}_{1 \perp}^{2}+m_{1}^{2}-i o}{p_{1}^{+}}\right)} \frac{\Lambda_{+}\left(p_{2 o n}\right)}{\left(K^{-}-p_{1}^{-}-\frac{\left(\vec{K}_{\perp}-\vec{p}_{1 \perp}\right)^{2}+m_{2}^{2}-i o}{K^{+}-p_{1}^{+}}\right)} \frac{1}{\left(p_{1}^{+}-k_{1}^{+}\right)} \\
& \times \frac{1}{\left(p_{1}^{-}-k_{1}^{-}-\frac{\left(\vec{p}_{1 \perp}-\vec{k}_{1 \perp}\right)^{2}+\mu^{2}-i o}{p_{1}^{+}-k_{1}^{+}}\right)} \frac{1}{k_{1}^{+}\left(K^{+}-k_{1}^{+}\right)} \frac{\Lambda_{+}\left(k_{1 o n}\right)}{\left(k_{1}^{-}-\frac{\vec{k}_{1 \perp}^{2}+m_{1}^{2}-i o}{k_{1}^{+}}\right)} \frac{\Lambda_{+}\left(k_{2 o n}\right)}{\left(K^{-}-k_{1}^{-}-\frac{\left(\vec{K}_{\perp}-\vec{k}_{1 \perp}\right)^{2}+m_{2}^{2}-i o}{K^{+}-k_{1}^{+}}\right)}
\end{aligned}
$$

The on-energy-shell values of the light-front minus momentum in Eq. (D4) are given in Eq. (C4), and

$$
\begin{gathered}
p_{1 o n}^{-}=\frac{\vec{p}_{1 \perp}^{2}+m_{1}^{2}}{p_{1}^{+}}, \\
p_{2 o n}^{-}=\frac{\left(\vec{K}_{\perp}-\vec{p}_{1 \perp}\right)^{2}+m_{2}^{2}}{K^{+}-p_{1}^{+}} .
\end{gathered}
$$


The matrix element $\left\langle k_{1}^{\prime+} \vec{k}_{1 \perp}^{\prime}\left\|\bar{G}_{0}(K) V(K) \bar{G}_{0}(K) V(K) \bar{G}_{0}(K)\right\| k_{1}^{+} \vec{k}_{1 \perp}\right\rangle$ is found by analytical integration in the light-front "energies" in Eq. (D4). To separate the intermediate four particle propagation, which occurs for $k_{11}^{+}, p_{1}^{+}$, and $k_{1}^{+}$satisfying $0<k_{1}^{+}<p_{1}^{+}<k^{\prime+}<K^{+}$, the following factorization is necessary:

$$
\begin{aligned}
& \frac{1}{K^{-}-p_{1}^{-}-\frac{\left(\vec{K}_{\perp}-\vec{p}_{1 \perp}\right)^{2}+m_{2}^{2}-i o}{K^{+}-p_{1}^{+}}} \frac{1}{p_{1}^{-}-k_{1}^{-}-\frac{\left(\vec{k}_{1 \perp}-\vec{p}_{1}\right)^{2}+\mu^{2}-i o}{p_{1}^{+}-k_{1}^{+}}} \\
& =\frac{1}{K^{-}-k_{1}^{-}-\frac{\left(\vec{K}_{\perp}-\vec{p}_{1 \perp}\right)^{2}+m_{2}^{2}-i o}{K^{+}-p_{1}^{+}}-\frac{\left(\vec{k}_{1 \perp}-\vec{p}_{1 \perp}\right)^{2}+\mu^{2}-i o}{p_{1}^{+}-k_{1}^{+}}} \\
& \times\left[\frac{1}{K^{-}-p_{1}^{-}-\frac{\left(\vec{K}_{\perp}-\vec{p}_{1 \perp}\right)^{2}+m_{2}^{2}-i o}{K^{+}-p_{1}^{+}}}+\frac{1}{p_{1}^{-}-k_{1}^{-}-\frac{\left(\vec{k}_{1 \perp}-\vec{p}_{1 \perp}\right)^{2}+\mu^{2}-i o}{p_{1}^{+}-k_{1}^{+}}}\right] \text {. }
\end{aligned}
$$

In all equations below, the convention is used that the positive energy spinor projectors with argument of the type ( $K$ $-k)_{o n}$ refer to the fermion labeled 2, while argument of the type $k_{o n}$ refers to the fermion labeled 1, if the fermion label is not explicit.

After the Cauchy integration in the light-front “energies," the result for $\left\langle k_{1}^{\prime+} \vec{k}_{1 \perp}^{\prime}\left\|\bar{G}_{0}(K) V(K) \bar{G}_{0}(K) V(K) \bar{G}_{0}(K)\right\| k_{1}^{+} \vec{k}_{1 \perp}\right\rangle$ in the region of $0<k_{1}^{+}<p_{1}^{+}<k_{1}^{\prime+}<K^{+}$, which is denoted by $\left\langle\left. k_{1}^{\prime+} \vec{k}_{1 \perp}^{\prime}|| \bar{G}_{0}(K) V(K) \bar{G}_{0}(K) V(K) \bar{G}_{0}(K)\right|_{(a)} \mid k_{1}^{+} \vec{k}_{1 \perp}\right\rangle$, is given by

$$
\begin{aligned}
& \left\langle k_{1}^{\prime+} \vec{k}_{1 \perp}^{\prime} \|\left.\bar{G}_{0}(K) V(K) \bar{G}_{0}(K) V(K) \bar{G}_{0}(K)\right|_{(a)} \mid k_{1}^{+} \vec{k}_{1 \perp}\right\rangle \\
& =\frac{\left(4 m_{1} m_{2}\right)^{3}\left(i g_{S}\right)^{4}}{2(2 \pi)^{3}} \int d p_{1}^{+} d^{2} p_{1 \perp} \frac{\theta\left(k_{1}^{\prime+}\right) \theta\left(K^{+}-k_{1}^{\prime+}\right)}{k_{1}^{\prime+}\left(K^{+}-k_{1}^{\prime+}\right)} \frac{i \Lambda_{+}\left(k_{1 o n}^{\prime}\right) \Lambda_{+}\left(\left(K-k_{1}^{\prime}\right)_{o n}\right)}{K^{-}-\frac{\vec{k}_{1 \perp}^{\prime}{ }^{2}+m_{1}^{2}}{k_{1}^{\prime+}}-\frac{\left(\vec{K}_{\perp}-\vec{k}_{1 \perp}^{\prime}\right)^{2}+m_{2}^{2}}{K^{+}-k_{1}^{\prime+}}+i o} \\
& \quad \times\left[F^{\prime}(K)+F^{\prime \prime}(K)\right] \frac{\theta\left(k_{1}^{+}\right) \theta\left(K^{+}-k_{1}^{+}\right)}{k_{1}^{+}\left(K^{+}-k_{1}^{+}\right)} \frac{i \Lambda_{+}\left(k_{1 o n}\right) \Lambda_{+}\left(\left(K-k_{1}\right)_{o n}\right)}{K^{-}-\frac{\vec{k}_{1 \perp}^{2}+m_{1}^{2}}{k_{1}^{+}}-\frac{\left(\vec{K}_{\perp}-\vec{k}_{1 \perp}\right)^{2}+m_{2}^{2}}{K^{+}-k_{1}^{+}}+i o},
\end{aligned}
$$

with

$$
\begin{aligned}
F^{\prime}(K)= & \frac{\theta\left(k_{1}^{\prime+}-p_{1}^{+}\right)}{\left(k_{1}^{\prime+}-p_{1}^{+}\right)} \frac{i \Lambda_{+}\left(p_{1 o n}\right) \Lambda_{+}\left(\left(K-k_{1}^{\prime}\right)_{o n}\right)}{K^{-}-\frac{\vec{p}_{1 \perp}^{2}+m_{1}^{2}}{p_{1}^{+}}-\frac{\left(\vec{K}_{\perp}-\vec{k}_{1 \perp}^{\prime}\right)^{2}+m_{2}^{2}}{K^{+}-k_{1}^{\prime+}}-\frac{\left(\vec{k}_{1 \perp}^{\prime}-\vec{p}_{1 \perp}\right)^{2}+\mu^{2}}{k_{1}^{\prime+}-p_{1}^{+}}+i o} \\
& \times \frac{\theta\left(p_{1}^{+}\right) \theta\left(K^{+}-p_{1}^{+}\right)}{p_{1}^{+}\left(K^{+}-p_{1}^{+}\right)} \frac{i \Lambda_{+}\left(p_{1 o n}\right) \Lambda_{+}\left(\left(K-p_{1}\right)_{o n}\right)}{K^{-}-\frac{\vec{p}_{1 \perp}^{2}+m_{1}^{2}}{p_{1}^{+}}-\frac{\left(\vec{K}_{\perp}-\vec{p}_{1 \perp}\right)^{2}+m_{2}^{2}}{K^{+}-p_{1}^{+}}+i o} \frac{\theta\left(p_{1}^{+}-k_{1}^{+}\right)}{\left(p_{1}^{+}-k_{1}^{+}\right)} \\
& \times \frac{i \Lambda_{+}\left(k_{1 o n}\right) \Lambda_{+}\left(\left(K-p_{1}\right)_{o n}\right)}{K^{-}-\frac{\vec{k}_{1 \perp}^{2}+m_{1}^{2}}{k_{1}^{+}}-\frac{\left(\vec{K}_{\perp}-\vec{p}_{1 \perp}\right)^{2}+m_{2}^{2}}{K^{+}-p_{1}^{+}}-\frac{\left(\vec{p}_{1 \perp}-\vec{k}_{1 \perp}\right)^{2}+\mu^{2}}{p_{1}^{+}-k_{1}^{+}}+i o},
\end{aligned}
$$




$$
\begin{aligned}
F^{\prime \prime}(K)= & \frac{\theta\left(k_{1}^{\prime+}-p_{1}^{+}\right)}{\left(k_{1}^{\prime+}-p_{1}^{+}\right)} \frac{i \Lambda_{+}\left(p_{1 o n}\right) \Lambda_{+}\left(\left(K-k_{1}^{\prime}\right)_{o n}\right)}{K^{-}-\frac{\vec{p}_{1 \perp}^{2}+m_{1}^{2}}{p_{1}^{+}}-\frac{\left(\vec{K}_{\perp}-\vec{k}_{1 \perp}^{\prime}\right)^{2}+m_{2}^{2}}{K^{+}-k_{1}^{\prime+}}-\frac{\left(\vec{k}_{1 \perp}^{\prime}-\vec{p}_{1 \perp}\right)^{2}+\mu^{2}}{k_{1}^{++}-p_{1}^{+}}+i o} \\
& \times \frac{i \Lambda_{+}\left(k_{1 o n}\right) \Lambda_{+}\left(\left(K-k_{1}^{\prime}\right)_{o n}\right)}{K^{-}-\frac{\vec{k}_{1 \perp}^{2}+m_{1}^{2}}{k_{1}^{+}}-\frac{\left(\vec{K}_{\perp}-\vec{k}_{1 \perp}^{\prime}\right)^{2}+m_{2}^{2}}{K^{+}-k_{1}^{\prime+}}-\frac{\left(\vec{k}_{1 \perp}^{\prime}-\vec{p}_{1 \perp}\right)^{2}+\mu^{2}}{k_{1}^{\prime+}-p_{1}^{+}}-\frac{\left(\vec{p}_{1 \perp}-\vec{k}_{1 \perp}\right)^{2}+\mu^{2}}{p_{1}^{+}-k_{1}^{+}}+i o} \\
& \times \frac{\theta\left(p_{1}^{+}\right) \theta\left(K^{+}-p_{1}^{+}\right)}{p_{1}^{+}\left(K^{+}-p_{1}^{+}\right)} \frac{\theta\left(p_{1}^{+}-k_{1}^{+}\right)}{\left(p_{1}^{+}-k_{1}^{+}\right)} \frac{i \Lambda_{+}\left(k_{1 o n}\right) \Lambda_{+}\left(\left(K-p_{1}\right)_{o n}\right)}{K^{-}-\frac{\vec{k}_{1 \perp}^{2}+m_{1}^{2}}{k_{1}^{+}}-\frac{\left(\vec{K}_{\perp}-\vec{p}_{1 \perp}\right)^{2}+m_{2}^{2}}{K^{+}-p_{1}^{+}}-\frac{\left(\vec{p}_{1 \perp}-\vec{k}_{1 \perp}\right)^{2}+\mu^{2}}{p_{1}^{+}-k_{1}^{+}}+i o} .
\end{aligned}
$$

The part of the propagator given by Eq. (D7) contains the virtual light-front propagation of intermediate states with up to four particles. The function $F^{\prime}$ contains only intermediate states with up to three particles and is two-body reducible. It will eventually be canceled by the corresponding piece in the second term in Eq. (D2). The function $F^{\prime \prime}$ has an intermediate state in which four-particles propagate that can be recognized as the middle piece of Eq. (D9). The other possibility which includes up to four particles in the intermediate state propagation is given by $0<k_{1}^{\prime+}<p_{1}^{+}<k_{1}^{+}<K^{+}$. To obtain this contribution, we exchange the external fermion states $1 \leftrightarrow 2$ in Eq. (D7).

The contribution of the region determined by $0<p_{1}^{+}<k_{1}^{+}<K^{+}$and $0<p_{1}^{+}<k_{1}^{\prime+}<K^{+}$to the matrix element $\left\langle k_{1}^{\prime}{ }_{1} \vec{k}_{1 \perp}^{\prime} \| \bar{G}_{0}(K) V(K) \bar{G}_{0}(K) V(K) \bar{G}_{0}(K)|| k_{1}^{+} \vec{k}_{1 \perp}\right\rangle$ is denoted by $\left\langle\left. k_{1}^{\prime+} \vec{k}_{1 \perp}^{\prime}|| \bar{G}_{0}(K) V(K) \bar{G}_{0}(K) V(K) \bar{G}_{0}(K)\right|_{(b)} \mid k_{1}^{+} \vec{k}_{1 \perp}\right\rangle$. It contains up to three-particle intermediate states only and is two-body reducible. Consequently, it will be canceled by the corresponding piece of the second term in Eq. (D2). It is given by

$$
\begin{aligned}
& \left\langle\left. k_{1}^{\prime+} \vec{k}_{1 \perp}^{\prime}|| \bar{G}_{0}(K) V(K) \bar{G}_{0}(K) V(K) \bar{G}_{0}(K)\right|_{(b)} \mid k_{1}^{+} \vec{k}_{1 \perp}\right\rangle \\
& =\frac{\left(4 m_{1} m_{2}\right)^{3}\left(i g_{S}\right)^{4}}{2(2 \pi)^{3}} \int d p_{1}^{+} d^{2} p_{1 \perp} \frac{\theta\left(k_{1}^{\prime+}\right) \theta\left(K^{+}-k_{1}^{\prime+}\right)}{k_{1}^{\prime+}\left(K^{+}-k_{1}^{\prime+}\right)} \frac{i \Lambda_{+}\left(k_{1 o n}^{\prime}\right) \Lambda_{+}\left(\left(K-k_{1}^{\prime}\right)_{o n}\right)}{K^{-}-\frac{\vec{k}_{1 \perp}^{\prime 2}+m_{1}^{2}}{k_{1}^{\prime+}}-\frac{\left(\vec{K}_{\perp}-k_{1 \perp}^{\prime}\right)^{2}+m_{2}^{2}}{K^{+}-k_{1}^{\prime+}}+i o} \\
& \times \frac{\theta\left(k_{1}^{\prime+}-k_{1}^{+}\right)}{\left(k_{1}^{\prime+}-k_{1}^{+}\right)} \frac{i \Lambda_{+}\left(p_{1 o n}\right) \Lambda_{+}\left(\left(K-k_{1}^{\prime}\right)_{o n}\right)}{K^{-}-\frac{\vec{p}_{1 \perp}^{2}+m_{1}^{2}}{p_{1}^{+}}-\frac{\left(\vec{K}_{\perp}-\vec{k}_{1 \perp}^{\prime}\right)^{2}+m_{2}^{2}}{K^{+}-k_{1}^{\prime+}}-\frac{\left(\vec{k}_{1 \perp}^{\prime}-\vec{p}_{1 \perp}\right)^{2}+\mu^{2}}{k_{1}^{\prime+}-p_{1}^{+}}+i o} \\
& \times \frac{\theta\left(p_{1}^{+}\right) \theta\left(K^{+}-p_{1}^{+}\right)}{p_{1}^{+}\left(K^{+}-p_{1}^{+}\right)} \frac{i \Lambda_{+}\left(p_{1 o n}\right) \Lambda_{+}\left(\left(K-p_{1}\right)_{o n}\right)}{K^{-}-\frac{\vec{p}_{1 \perp}^{2}+m_{1}^{2}}{p_{1}^{+}}-\frac{\left(\vec{K}_{\perp}-\vec{p}_{1 \perp}\right)^{2}+m_{2}^{2}}{K^{+}-p_{1}^{+}}+i o} \\
& \times \frac{\theta\left(k_{1}^{+}-p_{1}^{+}\right)}{\left(k_{1}^{+}-p_{1}^{+}\right)} \frac{i \Lambda_{+}\left(p_{1 o n}\right) \Lambda_{+}\left(\left(K-k_{1}^{\prime}\right)_{o n}\right)}{K^{-}-\frac{\vec{p}_{1 \perp}^{2}+m_{1}^{2}}{p_{1}^{+}}-\frac{\left(\vec{K}_{\perp}-\vec{k}_{1 \perp}^{\prime}\right)^{2}+m_{2}^{2}}{K^{+}-k_{1}^{\prime+}}-\frac{\left(\vec{k}_{1 \perp}^{\prime}-\vec{p}_{1 \perp}\right)^{2}+\mu^{2}}{k_{1}^{\prime+}-p_{1}^{+}}+i o}
\end{aligned}
$$

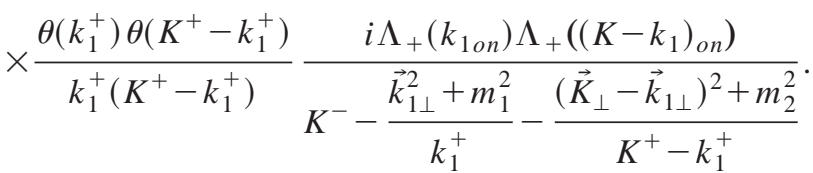

For the momentum region satisfying $0<k_{1}^{+}<p_{1}^{+}<K^{+}$and $0<k_{1}^{\prime+}<p_{1}^{+}<K^{+}$, the contribution to the matrix element $\left\langle k_{1}^{\prime+} \vec{k}_{1 \perp}^{\prime} \| \bar{G}_{0}(K) V(K) \bar{G}_{0}(K) V(K) \bar{G}_{0}(K)|| k_{1}^{+} \vec{k}_{1 \perp}\right\rangle$ can be obtained from Eq. (D10) by exchanging the variables of the fermions $1 \leftrightarrow 2$. From Eqs. (D9) and (D10), the following result is obtained: 


$$
\begin{aligned}
\left\langle k_{1}^{\prime+} \vec{k}_{1 \perp}^{\prime} \| \bar{G}_{0}(K) V(K) \bar{G}_{0}(K) V(K) \bar{G}_{0}(K)|| k_{1}^{+} \vec{k}_{1 \perp}\right\rangle= & \left(\left\langle\left. k_{1}^{\prime+} \vec{k}_{1 \perp}^{\prime}|| \bar{G}_{0}(K) V(K) \bar{G}_{0}(K) V(K) \bar{G}_{0}(K)\right|_{(a)} \mid k_{1}^{+} \vec{k}_{1 \perp}\right\rangle+[1 \leftrightarrow 2]\right) \\
& +\left(\left\langle\left. k_{1}^{\prime+} \vec{k}_{1 \perp}^{\prime}|| \bar{G}_{0}(K) V(K) \bar{G}_{0}(K) V(K) \bar{G}_{0}(K)\right|_{(b)} \mid k_{1}^{+} \vec{k}_{1 \perp}\right\rangle+[1 \leftrightarrow 2]\right) .
\end{aligned}
$$

The subtraction of the iterated first order driving term in Eq. (D2) cancels the corresponding terms in Eq. (D11) so that the matrix element, an operator in spinor space, $\left\langle k_{1}^{\prime+} \vec{k}_{1 \perp}^{\prime}\left|w_{\text {prop }}^{(4)}(K)\right| k_{1}^{+} \vec{k}_{1 \perp}\right\rangle$ is two-body irreducible and contains a global fourbody propagation. It is obtained from Eqs. (D7), (D9), and (D2) as

$$
\begin{aligned}
\left\langle k_{1}^{\prime+} \vec{k}_{1 \perp}^{\prime}\left|w_{\text {prop }}^{(4)}(K)\right| k_{1}^{+} \vec{k}_{1 \perp}\right\rangle & =\frac{2 m_{1} m_{2}\left(i g_{S}\right)^{4}}{(2 \pi)^{3}} \int d p_{1}^{+} d^{2} p_{1 \perp} \frac{\theta\left(k_{1}^{\prime+}-p_{1}^{+}\right)}{\left(k_{1}^{\prime+}-p_{1}^{+}\right)} \\
& \times \frac{i \Lambda_{+}\left(k_{1 o n}^{\prime}\right) \Lambda_{+}\left(p_{1 o n}\right) \Lambda_{+}\left(\left(K-k_{1}^{\prime}\right)_{o n}\right)}{K^{-}-\frac{\vec{p}_{1 \perp}^{2}+m_{1}^{2}}{p_{1}^{+}}-\frac{\left(\vec{K}_{\perp}-\vec{k}_{1 \perp}^{\prime}\right)^{2}+m_{2}^{2}}{K^{+}-k_{1}^{\prime+}}-\frac{\left(\vec{k}_{1 \perp}^{\prime}-\vec{p}_{1 \perp}\right)^{2}+\mu^{2}}{k_{1}^{\prime+}-p_{1}^{+}}+i o} \\
& \times \frac{\theta\left(p_{1}^{+}\right) \theta\left(K^{+}-p_{1}^{+}\right)}{p_{1}^{+}\left(K^{+}-p_{1}^{+}\right)} \frac{K^{-}-\frac{\vec{k}_{1 \perp}^{2}+m_{1}^{2}}{k_{1}^{+}}-\frac{\left(\vec{K}_{\perp}-\vec{k}_{1 \perp}^{\prime}\right)^{2}+m_{2}^{2}}{K^{+}-k_{+}^{\prime+}}-\frac{\left(\vec{k}_{1 \perp}^{\prime}-\vec{p}_{1 \perp}\right)^{2}+\mu^{2}}{k_{1}^{\prime+}-p_{1}^{+}}-\frac{\left(\vec{p}_{1 \perp}-\vec{k}_{1 \perp}\right)^{2}+\mu^{2}}{p_{1}^{+}-k_{1}^{+}}+i o}{i \Lambda_{+}\left(k_{1 o n}\right) \Lambda_{+}\left(\left(K-p_{1}\right)_{o n}\right) \Lambda_{+}\left(\left(K-k_{1}\right)_{o n}\right)} \\
& \times \frac{\theta\left(p_{1}^{+}-k_{1}^{+}\right)}{\left(p_{1}^{+}-k_{1}^{+}\right)} \frac{\vec{k}_{1 \perp}^{2}+m_{1}^{2}}{k_{1}^{+}}-\frac{\left(\vec{K}_{\perp}-\vec{p}_{1 \perp}\right)^{2}+m_{2}^{2}}{K^{+}-p_{1}^{+}}-\frac{\left(\vec{p}_{1 \perp}-\vec{k}_{1 \perp}\right)^{2}+\mu^{2}}{p_{1}^{+}-k_{1}^{+}}+i o
\end{aligned}
$$

Next, we discuss the contribution of the instantaneous terms of the Dirac propagators to the interaction $w^{(4)}$ derived from the operator $\left|\bar{G}_{0}(K) V(K) \Delta G_{0}^{F}(K) V(K) \bar{G}_{0}(K)\right|$. The integration over the $k^{-}$variable is performed in the above operator as it is done for Eq. (D4). Eight terms result, denoted by

$$
w_{\text {inst }}^{(4)}=\sum_{\alpha=1}^{8} w_{\text {inst }, \alpha}^{(4)} .
$$

The first one corresponds to the region of integration $0<k_{1}^{+}<p_{1}^{+}<k_{1}^{\prime+}<K^{+}$and the instantaneous term from fermion 1 . In this case, the bosons are absorbed by fermion 2:

$$
\begin{aligned}
\left\langle k_{1}^{\prime+} \vec{k}_{1 \perp}^{\prime}\left|w_{i n s t, 1}^{(4)}(K)\right| k_{1}^{+} \vec{k}_{1 \perp}\right\rangle= & \frac{m_{2}\left(i g_{S}\right)^{4}}{2(2 \pi)^{3}} \int d p_{1}^{+} d^{2} p_{1 \perp} \frac{\theta\left(k_{1}^{\prime+}-p_{1}^{+}\right)}{\left(k_{1}^{\prime+}-p_{1}^{+}\right)} \Lambda_{+}\left(k_{1 o n}^{\prime}\right) i \gamma_{1}^{+} \frac{\theta\left(p_{1}^{+}\right) \theta\left(K^{+}-p_{1}^{+}\right)}{p_{1}^{+}\left(K^{+}-p_{1}^{+}\right)} \\
& \times \frac{i \Lambda_{+}\left(k_{1 o n}\right) \Lambda_{+}\left(\left(K-k_{1}^{\prime}\right)_{o n}\right)}{\left(\vec{k}_{1 \perp}^{\prime}-\vec{p}_{1 \perp}\right)^{2}+\mu^{2}} \\
k_{1}^{\prime+}-p_{1}^{+} & \frac{\left(\vec{p}_{1 \perp}-\vec{k}_{1 \perp}\right)^{2}+\mu^{2}}{p_{1}^{+}-k_{1}^{+}}+i o \\
k_{1}^{+} & \frac{\left(\vec{K}_{\perp}-\vec{k}_{1 \perp}^{\prime}\right)^{2}+m_{2}^{2}}{K^{+}-k_{1}^{\prime+}}-\frac{\theta\left(p_{1}^{+}-k_{1}^{+}\right)}{\left(p_{1}^{+}-k_{1}^{+}\right)} \frac{\vec{k}_{+}\left(k_{1 o n}\right) \Lambda_{+}\left(\left(K-p_{1}\right)_{o n}\right) \Lambda_{+}\left(\left(K-k_{1}\right)_{o n}\right)}{K^{-}-\frac{\vec{k}_{1 \perp}^{2}+m_{1}^{2}}{k_{1}^{+}}-\frac{\left(\vec{K}_{\perp}-\vec{p}_{1 \perp}\right)^{2}+m_{2}^{2}}{K^{+}-p_{1}^{+}}-\frac{\left(\vec{p}_{1 \perp}-\vec{k}_{1 \perp}\right)^{2}+\mu^{2}}{p_{1}^{+}-k_{1}^{+}}+i o} .
\end{aligned}
$$

The contribution to the interaction, $w_{\text {inst }, 2}^{(4)}$, corresponds to the region of integration $0<k_{1}^{\prime+}<p_{1}^{+}<k_{1}^{+}<K^{+}$and the instantaneous term from fermion 2. It is obtained by exchanging fermions 1 and 2 in $w_{\text {inst }, 1}^{(4)}$ :

$$
\left\langle k_{1}^{\prime+} \vec{k}_{1 \perp}^{\prime}\left|w_{\text {inst }, 2}^{(4)}(K)\right| k_{1}^{+} \vec{k}_{1 \perp}\right\rangle=\left\langle k_{1}^{\prime+} \vec{k}_{1 \perp}^{\prime}\left|w_{\text {inst }, 1}^{(4)}(K)\right| k_{1}^{+} \vec{k}_{1 \perp}\right\rangle_{[1 \leftrightarrow 2]} .
$$


The third contribution corresponds to the region of integration of $0<k_{1}^{+}<p_{1}^{+}<k_{1}^{\prime+}<K^{+}$and the instantaneous term from fermion 1. The scalar bosons are emitted by fermion 2 ,

$$
\begin{aligned}
\left\langle k_{1}^{\prime+} \vec{k}_{1 \perp}^{\prime}\left|w_{i n s t, 3}^{(4)}(K)\right| k_{1}^{+} \vec{k}_{1 \perp}\right\rangle & =\frac{m_{2}\left(i g_{S}\right)^{4}}{2(2 \pi)^{3}} \int d p_{1}^{+} d^{2} p_{1 \perp} \frac{\theta\left(k_{1}^{\prime+}-p_{1}^{+}\right)}{\left(k_{1}^{\prime+}-p_{1}^{+}\right)} \frac{i \Lambda_{+}\left(k_{1 o n}^{\prime}\right) \Lambda_{+}\left(p_{1 o n}\right) \Lambda_{+}\left(\left(K-k_{1}^{\prime}\right)_{o n}\right)}{K^{-}-\frac{\vec{p}_{1 \perp}^{2}+m_{1}^{2}}{p_{1}^{+}}-\frac{\left(\vec{K}_{\perp}-\vec{k}_{1 \perp}^{\prime}\right)^{2}+m_{2}^{2}}{K^{+}-k_{1}^{\prime+}}-\frac{\left(\vec{k}_{1 \perp}^{\prime}-\vec{p}_{1 \perp}\right)^{2}+\mu^{2}}{k_{1}^{\prime+}-p_{1}^{+}}+i o} \\
& \times \frac{\theta\left(p_{1}^{+}\right) \theta\left(K^{+}-p_{1}^{+}\right)}{p_{1}^{+}\left(K^{+}-p_{1}^{+}\right)} \frac{i \Lambda_{+}\left(\left(K-k_{1}^{\prime}\right)_{o n}\right) \Lambda_{+}\left(k_{1 o n}\right)}{K^{-}-\frac{\vec{k}_{1 \perp}^{2}+m_{1}^{2}}{k_{1}^{+}}-\frac{\left(\vec{K}_{\perp}-\vec{k}_{1 \perp}^{\prime}\right)^{2}+m_{2}^{2}}{K^{+}-k_{1}^{\prime+}}-\frac{\left(\vec{k}_{1 \perp}^{\prime}-\vec{p}_{1 \perp}\right)^{2}+\mu^{2}}{k_{1}^{\prime+}-p_{1}^{+}}-\frac{\left(\vec{p}_{1 \perp}-\vec{k}_{1 \perp}\right)^{2}+\mu^{2}}{p_{1}^{+}-k_{1}^{+}}+i o} \\
& \times \frac{\theta\left(p_{1}^{+}-k_{1}^{+}\right)}{\left(p_{1}^{+}-k_{1}^{+}\right)} i \gamma_{2}^{+} \Lambda_{+}\left(\left(K-k_{1}\right)_{o n}\right) .
\end{aligned}
$$

The contribution to the interaction, $w_{\text {inst } 4}^{(4)}$, corresponds to the region of integration of $0<k_{1}^{\prime+}<p_{1}^{+}<k_{1}^{+}<K^{+}$and the instantaneous term from fermion 1 . It is obtained by exchanging fermions 1 and 2 in $w_{\text {inst }, 3}^{(4)}$ :

$$
\left\langle k_{1}^{\prime+} \vec{k}_{1 \perp}^{\prime}\left|w_{\text {inst }, 4}^{(4)}(K)\right| k_{1}^{+} \vec{k}_{1 \perp}\right\rangle=\left\langle k_{1}^{\prime+} \vec{k}_{1 \perp}^{\prime}\left|w_{\text {inst }, 3}^{(4)}(K)\right| k_{1}^{+} \vec{k}_{1 \perp}\right\rangle_{[1 \leftrightarrow 2]}
$$

The contribution to the interaction, $w_{\text {inst }, 5}^{(4)}$, corresponds to the region of integration that satisfies $0<p_{1}^{+}<k_{1}^{\prime+}<K^{+}$and $0<p_{1}^{+}<k_{1}^{+}<K^{+}$. The instantaneous term comes from fermion 2 . The boson is emitted by the initial fermion 1 , absorbed by fermion 2 and reemitted instantaneously by it and absorbed by fermion 1 :

$$
\begin{aligned}
\left\langle k_{1}^{\prime+} \vec{k}_{1 \perp}^{\prime}\left|w_{\text {inst }, 5}^{(4)}(K)\right| k_{1}^{+} \vec{k}_{1 \perp}\right\rangle & =\frac{m_{1}\left(i g_{S}\right)^{4}}{2(2 \pi)^{3}} \int d p_{1}^{+} d^{2} p_{1 \perp} \frac{\theta\left(k_{1}^{\prime+}-k_{1}^{+}\right)}{\left(k_{1}^{\prime+}-k_{1}^{+}\right)} \frac{i \Lambda_{+}\left(k_{1 o n}^{\prime}\right) \Lambda_{+}\left(p_{1 o n}\right) \Lambda_{+}\left(\left(K-k_{1}^{\prime}\right)_{o n}\right)}{K^{-}-\frac{\vec{p}_{1 \perp}^{2}+m_{1}^{2}}{p_{1}^{+}}-\frac{\left(\vec{K}_{\perp}-\vec{k}_{1 \perp}^{\prime}\right)^{2}+m_{2}^{2}}{K^{+}-k_{1}^{\prime+}}-\frac{\left(\vec{k}_{1 \perp}^{\prime}-\vec{p}_{1 \perp}\right)^{2}+\mu^{2}}{k_{1}^{\prime+}-p_{1}^{+}}+i o} \\
& \times \frac{\theta\left(p_{1}^{+}\right) \theta\left(K^{+}-p_{1}^{+}\right)}{p_{1}^{+}\left(K^{+}-p_{1}^{+}\right)} i \gamma_{2}^{+} \frac{\theta\left(k_{1}^{+}-p_{1}^{+}\right)}{\left(k_{1}^{+}-p_{1}^{+}\right)} \frac{i \Lambda_{+}\left(p_{1 o n}\right) \Lambda_{+}\left(\left(K-k_{1}\right) \text { on }\right) \Lambda_{+}\left(k_{1 o n}\right)}{K^{-}-\frac{\vec{p}_{1 \perp}^{2}+m_{1}^{2}}{p_{1}^{+}}-\frac{\left(\vec{K}_{\perp}-\vec{k}_{1 \perp}\right)^{2}+m_{2}^{2}}{K^{+}-k_{1 \perp}^{+}}-\frac{\left.\vec{p}_{1 \perp}\right)^{2}+\mu^{2}}{k_{1}^{+}-p_{1}^{+}}+i o} .
\end{aligned}
$$

The contribution to the interaction, $w_{\text {inst, } 6}^{(4)}$, corresponds to the region of integration that satisfies $0<k_{1}^{\prime+}<p_{1}^{+}<K^{+}$and $0<k_{1}^{+}<p_{1}^{+}<K^{+}$. The instantaneous term comes from fermion 1 . The boson is emitted by the initial fermion 2 , absorbed by fermion 1 and reemitted instantaneously by it and absorbed by fermion 2 . It is obtained by exchanging fermions 1 and 2 in $w_{\text {inst }, 5}^{(4)}$,

$$
\left\langle k_{1}^{\prime+} \vec{k}_{1 \perp}^{\prime}\left|w_{\text {inst }, 6}^{(4)}(K)\right| k_{1}^{+} \vec{k}_{1 \perp}\right\rangle=\left\langle k_{1}^{\prime+} \vec{k}_{1 \perp}^{\prime}\left|w_{\text {inst }, 5}^{(4)}(K)\right| k_{1}^{+} \vec{k}_{1 \perp}\right\rangle_{[1 \leftrightarrow 2]}
$$

The contribution to the interaction, $w_{i n s t, 7}^{(4)}$, corresponds to the region of integration of $0<k_{1}^{+}<p_{1}^{+}<k_{1}^{\prime+}<K^{+}$and the instantaneous terms from both fermions 1 and 2. In this case, the bosons are emitted by fermion 1 and absorbed by fermion 2 ,

$$
\begin{aligned}
\left\langle k_{1}^{\prime+} \vec{k}_{1 \perp}^{\prime}\left|w_{\text {inst }, 7}^{(4)}(K)\right| k_{1}^{+} \vec{k}_{1 \perp}\right\rangle & \\
= & \frac{\left(i g_{S}\right)^{4}}{4(2 \pi)^{3}} \int d p_{1}^{+} d^{2} p_{1 \perp} \frac{\theta\left(k_{1}^{\prime+}-p_{1}^{+}\right)}{\left(k_{1}^{\prime+}-p_{1}^{+}\right)} \Lambda_{+}\left(k_{1 o n}^{\prime}\right) i \gamma_{1}^{+} \frac{\theta\left(p_{1}^{+}\right) \theta\left(K^{+}-p_{1}^{+}\right)}{p_{1}^{+}\left(K^{+}-p_{1}^{+}\right)} \\
& \left.\times \frac{i \Lambda_{+}\left(\left(K-k_{1}^{\prime}\right)_{o n}\right) \Lambda_{+}\left(k_{1 o n}\right)}{k_{1}^{+}}-\frac{\left(\vec{K}_{\perp}-\vec{k}_{1 \perp}^{\prime}\right)^{2}+m_{2}^{2}}{K^{+}-k_{1}^{\prime+}}-\frac{\left(\vec{k}_{1 \perp}^{\prime}-\vec{p}_{1 \perp}\right)^{2}+\mu^{2}}{k_{1}^{\prime+}-p_{1}^{+}}-\frac{\left(\vec{p}_{1 \perp}-\vec{k}_{1 \perp}\right)^{2}+\mu^{2}}{p_{1}^{+}-k_{1}^{+}}+i m_{1}^{+}-k_{1}^{+}\right) \\
& K^{-}-\frac{k_{1}^{+} \Lambda_{+}}{\left(\left(p_{1}^{+}-k_{1}\right)_{o n}\right) .}
\end{aligned}
$$


The contribution to the interaction, $w_{\text {inst }, 8}^{(4)}$, corresponds to the region of integration of $0<k_{1}^{\prime+}<p_{1}^{+}<k_{1}^{+}<K^{+}$and the instantaneous terms from both fermions 1 and 2 . It is obtained by exchanging fermions 1 and 2 in $w_{\text {inst }, 7}^{(4)}$ :

$$
\left\langle k_{1}^{\prime+} \vec{k}_{1 \perp}^{\prime}\left|w_{\text {inst }, 8}^{(4)}(K)\right| k_{1}^{+} \vec{k}_{1 \perp}\right\rangle=\left\langle k_{1}^{\prime+} \vec{k}_{1 \perp}^{\prime}\left|w_{\text {inst }, 7}^{(4)}(K)\right| k_{1}^{+} \vec{k}_{1 \perp}\right\rangle_{[1 \leftrightarrow 2]} .
$$

\section{APPENDIX E: INTEGRAL EQUATION FOR THE BOUND STATE}

In the approximation considered, the vertex function satisfies an integral equation with the kernel containing two parts, one corresponding to Eq. (C5) and the other to Eq. (D2). The plus momentum are rescaled by $K^{+}$, such that the momentum fractions $x=k_{1}^{+} / K^{+}, y=k_{1}^{\prime+} / K^{+}$, and $z=p_{1}^{+} / K^{+}$, are used. The notation $\left\langle k_{1}^{\prime+} \vec{k}_{1 \perp}^{\prime} \mid \gamma_{B}\right\rangle \equiv \gamma_{B}\left(y, \vec{k}_{1 \perp}^{\prime}\right)$ will be used. The homogeneous integral equation for the light-front vertex function is evaluated in the center-of-mass system, is

$$
\gamma_{B}\left(y, \vec{k}_{1 \perp}^{\prime}\right)=\frac{1}{(2 \pi)^{3}} \int \frac{d^{2} k_{1 \perp} d x}{2 x(1-x)} \frac{\mathcal{K}^{(2)}\left(y, \vec{k}_{1 \perp}^{\prime} ; x, \vec{k}_{1 \perp}\right)+\mathcal{K}^{(4)}\left(y, \vec{k}_{1 \perp}^{\prime} ; x, \vec{k}_{1 \perp}\right)}{M_{B}^{2}-M_{0}^{2}} \gamma_{B}\left(x, \vec{k}_{1 \perp}\right),
$$

where the free two-body mass is $M_{0}^{2}=\left(\vec{k}_{1 \perp}^{2}+m^{2}\right) / x(1-x)$ and $0<x<1$. The effective interaction is defined according to $\mathcal{K}^{(n)}\left(y, \vec{k}_{1 \perp}^{\prime} ; x, \vec{k}_{1 \perp}\right)=i\left\langle k_{1}^{\prime+} \vec{k}_{1 \perp}^{\prime}\left|w^{(n)}\left(K_{B}\right)\right| k_{1}^{+} \vec{k}_{1 \perp}\right\rangle$.

The part of the kernel which includes only the propagation of virtual three particles states forward in the light-front time is obtained from Eq. (C5). In all equations below, the positive energy spinor projectors with argument of the type $(K-k)_{\text {on }}$ refer to the fermion labeled 2, while argument of the type $k_{\text {on }}$ refers to the fermion labeled 1 . We have

$$
\begin{aligned}
\mathcal{K}^{(2)}\left(y, \vec{k}_{1 \perp}^{\prime} ; x, \vec{k}_{1 \perp}\right)= & \Lambda_{+}\left(k_{1 o n}^{\prime}\right) \Lambda_{+}\left(k_{1 o n}\right) \Lambda_{+}\left(\left(K-k_{1}^{\prime}\right)_{o n}\right) \Lambda_{+}\left(\left(K-k_{1}\right)_{o n}\right) g_{S}^{2} \\
& \times \frac{\theta(y-x)}{(x-y)\left(M_{B}^{2}-\frac{\vec{k}_{1 \perp}^{\prime 2}+m^{2}}{1-y}-\frac{\vec{k}_{1 \perp}^{2}+m^{2}}{x}-\frac{\left(\vec{k}_{1 \perp}^{\prime}-\vec{k}_{1 \perp}\right)^{2}+\mu^{2}}{y-x}\right)}+[1 \leftrightarrow 2] .
\end{aligned}
$$

Equation (E1) with the effective interaction given by Eq. (E2) corresponds to the fermionic version of the bosonic Weinberg equation derived from the BSE in the infinitum momentum frame [2]. Other works have discussed the two-boson bound state in the one-boson-exchange approximation [19] and also including self-energy corrections [20].

The contribution to the kernel from the interaction evaluated at order $g_{S}^{4}$ has two terms, $\mathcal{K}^{(4)}=\mathcal{K}_{\text {prop }}^{(4)}+\mathcal{K}_{\text {inst }}^{(4)}$, one that comes from the propagating part of the Dirac propagators, $\mathcal{K}_{\text {prop }}^{(4)}$ and another that comes from the contribution of the instantaneous terms, $\mathcal{K}_{\text {inst }}^{(4)}$. The term $\mathcal{K}_{\text {prop }}^{(4)}$ coming from the virtual four-body propagation, is obtained from Eq. (D12) as

$$
\begin{aligned}
\mathcal{K}_{p r o p}^{(4)}\left(y, \vec{k}_{1 \perp}^{\prime} ; x, \vec{k}_{1 \perp}\right)= & \frac{4 m^{2} g_{S}^{4}}{(2 \pi)^{3}} \int \frac{d^{2} p_{1 \perp} d z}{2 z(1-z)(z-x)(y-z)} \frac{\theta(y-z) \theta(z-x) \Lambda_{+}\left(k_{1 o n}^{\prime}\right) \Lambda_{+}\left(\left(K-k_{1}^{\prime}\right)_{o n}\right)}{\left(M_{B}^{2}-\frac{\vec{k}_{1 \perp}^{\prime 2}+m^{2}}{1-y}-\frac{\vec{p}_{1 \perp}^{2}+m^{2}}{z}-\frac{\left(\vec{k}_{1 \perp}^{\prime}-\vec{p}_{1 \perp}\right)^{2}+\mu^{2}}{y-z}\right)} \\
& \times \frac{\Lambda_{+}\left(p_{1 o n}\right) \Lambda_{+}\left(\left(K-p_{1}\right)_{o n}\right)}{\left(M_{B}^{2}-\frac{\vec{k}_{1 \perp}^{\prime 2}+m^{2}}{1-y}-\frac{\vec{k}_{1 \perp}^{2}+m^{2}}{x}-\frac{\left(\vec{k}_{1 \perp}^{\prime}-\vec{p}_{1 \perp}\right)^{2}+\mu^{2}}{y-z}-\frac{\left(\vec{p}_{1 \perp}-\vec{k}_{1 \perp}\right)^{2}+\mu^{2}}{z-x}\right)} \\
& \times \frac{\Lambda_{+}\left(k_{1 o n}\right) \Lambda_{+}\left(\left(K-k_{1}\right)_{o n}\right)}{\left(M_{B}^{2}-\frac{\vec{p}_{1 \perp}^{2}+m^{2}}{1-z}-\frac{\vec{k}_{1 \perp}^{2}+m^{2}}{x}-\frac{\left(\vec{p}_{1 \perp}-\vec{k}_{1 \perp}\right)^{2}+\mu^{2}}{z-x}\right)}+[1 \leftrightarrow 2] .
\end{aligned}
$$

The term $\mathcal{K}_{\text {inst }}^{(4)}$ coming from the instantaneous terms of the Dirac propagators, is obtained from Eqs. (D14)-(D21). The eight terms that result are denoted by

$$
\mathcal{K}_{\text {inst }}^{(4)}=\sum_{\alpha=1}^{8} \mathcal{K}_{\text {inst }, \alpha}^{(4)}
$$

The terms $\mathcal{K}_{\text {inst } 1}^{(4)}+\mathcal{K}_{\text {inst }, 2}^{(4)}$ are derived from Eqs. (D14) and (D15), through the substitution of the ratios $k_{1}^{+} / K^{+}, k^{\prime}$ $+{ }_{1} / K^{+}$, and $p_{1}^{+} / K^{+}$by $x, y$, and $z$, respectively: 


$$
\begin{aligned}
\mathcal{K}_{i n s t, 1}^{(4)}\left(y, \vec{k}_{1 \perp}^{\prime} ; x, \vec{k}_{1 \perp}\right)+\mathcal{K}_{i n s t, 2}^{(4)}\left(y, \vec{k}_{1 \perp}^{\prime} ; x, \vec{k}_{1 \perp}\right)= & \frac{m g_{S}^{4}}{(2 \pi)^{3}} \int \frac{d^{2} p_{1 \perp} d z}{2 z(1-z)(z-x)(y-z)} \theta(y-z) \theta(z-x) \Lambda_{+}\left(k_{1 o n}^{\prime}\right) \gamma_{1}^{+} \Lambda_{+}\left(\left(K-k_{1}^{\prime}\right)_{o n}\right) \\
& \times \frac{\Lambda_{+}\left(p_{1 o n}\right) \Lambda_{+}\left(\left(K-p_{1}\right)_{o n}\right)}{\left(M_{B}^{2}-\frac{\vec{k}_{1 \perp}^{\prime 2}+m^{2}}{1-y}-\frac{\vec{k}_{1 \perp}^{2}+m^{2}}{x}-\frac{\left(\vec{k}_{1 \perp}^{\prime}-\vec{p}_{1 \perp}\right)^{2}+\mu^{2}}{y-z}-\frac{\left(\vec{p}_{1 \perp}-\vec{k}_{1 \perp}\right)^{2}+\mu^{2}}{z-x}\right)} \\
& \times \frac{\Lambda_{+}\left(k_{1 o n}\right) \Lambda_{+}\left(\left(K-k_{1}\right)_{o n}\right)}{\left(M_{B}^{2}-\frac{\vec{p}_{1 \perp}^{2}+m^{2}}{1-z}-\frac{\vec{k}_{1 \perp}^{2}+m^{2}}{x}-\frac{\left(\vec{p}_{1 \perp}\right)^{2}+\mu^{2}}{z-x}\right)}+[1 \leftrightarrow 2] .
\end{aligned}
$$

The contribution to the kernel, $\mathcal{K}_{\text {inst }, 3}^{(4)}+\mathcal{K}_{\text {inst }, 4}^{(4)}$ comes from Eqs. (D16) and (D17),

$\mathcal{K}_{i n s t, 3}^{(4)}\left(y, \vec{k}_{1 \perp}^{\prime} ; x, \vec{k}_{1 \perp}\right)+\mathcal{K}_{i n s t, 4}^{(4)}\left(y, \vec{k}_{1 \perp}^{\prime} ; x, \vec{k}_{1 \perp}\right)=\frac{m g_{S}^{4}}{(2 \pi)^{3}} \int \frac{d^{2} p_{1 \perp} d z}{2 z(1-z)(z-x)(y-z)}$

$$
\begin{aligned}
& \times \frac{\theta(y-z) \theta(z-x) \Lambda_{+}\left(k_{1 \text { on }}^{\prime}\right) \Lambda_{+}\left(\left(K-k_{1}^{\prime}\right)_{o n}\right)}{\left(M_{B}^{2}-\frac{\vec{k}_{1 \perp}^{\prime 2}+m^{2}}{1-y}-\frac{\vec{p}_{1 \perp}^{2}+m^{2}}{z}-\frac{\left(\vec{k}_{1 \perp}^{\prime}-\vec{p}_{1 \perp}\right)^{2}+\mu^{2}}{y-z}\right)} \\
& \times \frac{\Lambda_{+}\left(p_{1 \text { on }}\right) \Lambda_{+}\left(\left(K-p_{1}\right)_{o n}\right)}{\left(M_{B}^{2}-\frac{\vec{k}_{1 \perp}^{\prime 2}+m^{2}}{1-y}-\frac{\vec{k}_{1 \perp}^{2}+m^{2}}{x}-\frac{\left(\vec{k}_{1 \perp}^{\prime}-\vec{p}_{1 \perp}\right)^{2}+\mu^{2}}{y-z}-\frac{\left(\vec{p}_{1 \perp}-\vec{k}_{1 \perp}\right)^{2}+\mu^{2}}{z-x}\right)} \\
& \times \Lambda_{+}\left(k_{1 \text { on }}\right) \gamma_{2}^{+} \Lambda_{+}\left(\left(K-k_{1}\right)_{o n}\right)+[1 \leftrightarrow 2] .
\end{aligned}
$$

The contribution to the kernel of Eq. $(\mathrm{E} 1), \mathcal{K}_{\text {inst }, 5}^{(4)}+\mathcal{K}_{\text {inst }, 6}^{(4)}$, comes from Eqs. (D18) and (D19):

$$
\begin{aligned}
\mathcal{K}_{i n s t, 5}^{(4)}\left(y, \vec{k}_{1 \perp}^{\prime} ; x, \vec{k}_{1 \perp}\right)+\mathcal{K}_{i n s t, 6}^{(4)}\left(y, \vec{k}_{1 \perp}^{\prime} ; x, \vec{k}_{1 \perp}\right)= & \frac{2 m g_{S}^{4}}{(2 \pi)^{3}} \int \frac{d^{2} p_{1 \perp} d z}{2 z(1-z)(z-x)(y-z)} \\
& \times \frac{\theta(y-z) \theta(x-z) \Lambda_{+}\left(k_{1 o n}^{\prime}\right) \Lambda_{+}\left(\left(K-k_{1}^{\prime}\right)_{o n}\right)}{\left(M_{B}^{2}-\frac{\vec{k}_{1 \perp}^{\prime 2}+m^{2}}{1-y}-\frac{\vec{p}_{1 \perp}^{2}+m^{2}}{z}-\frac{\left(\vec{k}_{1 \perp}^{\prime}-\vec{p}_{1 \perp}\right)^{2}+\mu^{2}}{y-z}\right)} \Lambda_{+}\left(p_{1 o n}\right) \gamma_{2}^{+} \\
& \left.\times \frac{\Lambda_{+}\left(k_{1 o n}\right) \Lambda_{+}\left(\left(K-k_{1}\right)_{o n}\right)}{\left(M_{B}^{2}-\frac{\vec{p}_{1 \perp}^{2}+m^{2}}{z}-\frac{\vec{k}_{1 \perp}^{2}+m^{2}}{1-x}-\frac{\left.\vec{k}_{1 \perp}\right)^{2}+\mu^{2}}{x-z}+[1 \leftrightarrow 2]\right.}\right)
\end{aligned}
$$

The contribution to the kernel of Eq. $(\mathrm{E} 1), \mathcal{K}_{\text {inst }, 7}^{(4)}+\mathcal{K}_{\text {inst }, 8}^{(4)}$, comes from Eqs. (D20) and (D21):

$$
\begin{aligned}
\mathcal{K}_{i n s t, 7}^{(4)}\left(y, \vec{k}_{1 \perp}^{\prime} ; x, \vec{k}_{1 \perp}\right)+\mathcal{K}_{i n s t, 8}^{(4)}\left(y, \vec{k}_{1 \perp}^{\prime} ; x, \vec{k}_{1 \perp}\right)= & \frac{g_{S}^{4}}{2(2 \pi)^{3}} \int \frac{d^{2} p_{1 \perp} d z}{2 z(1-z)(z-x)(y-z)} \\
& \times \theta(y-z) \theta(z-x) \Lambda_{+}\left(k_{1 o n}^{\prime}\right) \Lambda_{+}\left(\left(K-k_{1}^{\prime}\right)_{o n}\right) \gamma_{1}^{+} \\
& \times \frac{\Lambda_{+}\left(p_{1 o n}\right) \Lambda_{+}\left(\left(K-p_{1}\right)_{o n}\right)}{\left(M_{B}^{2}-\frac{\vec{k}_{1 \perp}^{\prime 2}+m^{2}}{1-y}-\frac{\vec{k}_{1 \perp}^{2}+m^{2}}{x}-\frac{\left(\vec{k}_{1 \perp}^{\prime}-\vec{p}_{1 \perp}\right)^{2}+\mu^{2}}{y-z}-\frac{\left(\vec{p}_{1 \perp}-\vec{k}_{1 \perp}\right)^{2}+\mu^{2}}{z-x}\right)} \\
& \times \Lambda_{+}\left(k_{1 o n}\right) \gamma_{2}^{+} \Lambda_{+}\left(\left(K-k_{1}\right)_{o n}\right)+[1 \leftrightarrow 2] .
\end{aligned}
$$


Equations (E1)-(E8) are easily recognized to be covariant under kinematical light-front boosts. However, the covariance of the four-dimensional wave function, Eq. (B2), is certainly lost by the finite expansion of $W(K)$, given in Eq. (16), and the use of the corresponding $w(K)$. Covariance continues to hold however for the solution of Eq. (16).

[1] E. E. Salpeter and H. A. Bethe, Phys. Rev. 84, 1232 (1951).

[2] S. Weinberg, Phys. Rev. 150, 1313 (1966).

[3] L. L. Frankfurt and M. I. Strikman, Nucl. Phys. B148, 107 (1979); Phys. Rep. 76, 215 (1981).

[4] R. J. Perry, A. Harindranath, and K. G. Wilson, Phys. Rev. Lett. 65, 2959 (1990).

[5] S. Glasek, A. Harindranath, S. Pinsky, J. Shigemitsu, and K. G. Wilson, Phys. Rev. D 47, 1599 (1993).

[6] S. J. Brodsky, H.-C. Pauli, and S. Pinsky, Phys. Rep. 301, 299 (1998).

[7] J. Carbonell, B. Desplanques, V. A. Karmanov, and J. F. Mathiot, Phys. Rep. 300, 215 (1998).

[8] J. H. O. Sales, T. Frederico, B. V. Carlson, and P. U. Sauer, Phys. Rev. C 61, 044003 (2000).

[9] J. R. Cooke, G. A. Miller, and D. R. Phillips, Phys. Rev. C 61, 064005 (2000); J. R. Cooke and G. A. Miller, ibid. 62, 054008 (2000).

[10] M. Mangin-Brinet and J. Carbonell, Phys. Lett. B 474, 237
(2000).

[11] N. C. J. Schoonderwoerd, B. L. G. Bakker, and V. A. Karmanov, Phys. Rev. C 58, 3093 (1998).

[12] M. G. Fuda and Y. Zhang, Phys. Rev. C 51, 23 (1995); 52, 1260 (1995)

[13] P. G. Blunden, M. Burkardt, and G. A. Miller, Phys. Rev. C 59, 2998 (1999); 60, 055211 (1999).

[14] G. A. Miller and R. Machleidt, Phys. Lett. B 455, 19 (1999); Phys. Rev. C 60, 035202 (1999).

[15] P. M. Wort, Phys. Rev. D 47, 608 (1993).

[16] R. M. Woloshyn and A. D. Jackson, Nucl. Phys. B64, 269 (1973).

[17] C. Itzykson and J.-B. Zuber, Quantum Field Theory (McGrawHill, International Edition, New York, 1985).

[18] P. A. M. Dirac, Rev. Mod. Phys. 21, 392 (1949).

[19] C.-R. Ji and R. J. Furnstahl, Phys. Lett. 167B, 11 (1986); C.-R. Ji, ibid. 167B, 16 (1986).

[20] C.-R. Ji, Phys. Lett. B 322, 389 (1994). 